\title{
Integrals, Jump-Diffusion Processes and Monte Carlo Simulation
}

by

Yue Huang

\begin{abstract}
A thesis submitted to the Faculty of Graduate and Postdoctoral Affairs
in partial fulfillment of the requirements for the degree of

Master of Science

in

Probability and Statistics

Carleton University

Ottawa, Ontario
\end{abstract}

@ 2013

Yue Huang 


\section{Acknowledgements}

I want to give my special appreciation to my supervisors Dr. Donald Dawson and Dr. Yiqiang Zhao, for their gentleness, their selfless warm help and encouragement, which touch and ignite my passion yet remembering to be humble and thankful in pursuing the scientific spirit. I would also like to give thanks to my parents, for their support, always almost anytime. 


\section{Abstract}

This master thesis is to fulfill the curiosity in how special characteristics of Brownian motion motivate and distinguish the development of Itô calculus from the close relative - Stieltjes integral. Therefore, firstly we will see the general Stieltjes integral theories and how the concept of $p$-variation plays a determining role in Stieltjes integrability and the case where the fractional Brownian motion can also be an integrator given certain Hurst parameters under the Stieltjes scope in terms of Young's extension of $p$-variation theory. And then we will give a tour of the distinctive traits of Brownian motion including its property on $p$-variation as well as the Big-3 properties, namely, nowhere differentiability, unbounded variation, positive quadratic variation and how they mark the necessity to evolve a new Itô integral on integrator Brownian motion from the standard Stieltjes integral. Subsequently, we expand the integrator in Itô integral from Brownian motion with continuous path to Itô integral as a continuous diffusion process and further to jump-diffusion processes. Thereafter, we see how these new stochastic processes, all of which, results of the newly defined stochastic integrations, play a role in modelling financial assets as stochastic differential equations and in the end how sample paths of these stochastic processes can be generated using simulation methods and examplify the fact that Jump-Diffusion models are improvements from classical Black-Scholes-Merton model to incorporate the fat tail effects usually exhibited in empricial financial data. 


\section{Contents}

Acknowledgements

Abstract

\begin{tabular}{lll}
\hline & Introduction & 1
\end{tabular}

1.1 Gaussian Process . . . . . . . . . . . . . . . . . . . . 3

1.2 Brownian Motion $\ldots \ldots \ldots \ldots$

1.3 Fractional Brownian Motion $(\mathrm{fBm}) \ldots \ldots$

1.3.1 Comparison with standard Brownian motion . . . . . . . . . . 4

1.3.2 Properties in terms of $\mathrm{H}:$. . . . . . . . . . . . . . . 4

1.3.3 Other Properties: . . . . . . . . . . . . . . . . . 4

1.3.4 Applications: . . . . . . . . . . . . . . . . 4 4

1.4 Itô Process . . . . . . . . . . . . . . . . . . . . . 5

1.5 Jump Processes $\ldots \ldots \ldots \ldots$

1.5.1 Pure Jump Process

- the Building Block of Jump Stochastic Integrals . . . . . . . . 5

1.5.2 General Jump Process . . . . . . . . . . . . . . . 8

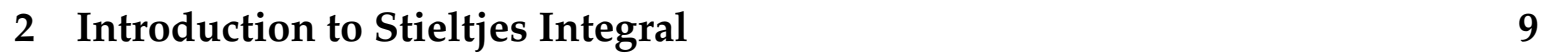

2.1 Definitions and Notations . . . . . . . . . . . . . . . 9

2.2 Theorems on Upper and Lower Stieltjes Sums . . . . . . . . . . . . . 10

2.3 Properties of Stieltjes Integrable Function . . . . . . . . . . . . . . . . 11

2.4 Sufficient Conditions to Determine Stieltjes Integrability . . . . . . . 11

2.4 .1 Theorems to Determine Stieltjes Integrability . . . . . . . . . . 12

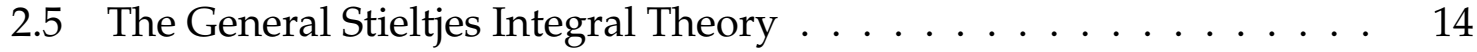

2.5.1 Sufficient Conditions of the Existence of a Stieltjes Integral . . 14 
$\begin{array}{lll}3 & \text { Stieltjes Integral with Fractional Brownian Motion as the Integrator } & 18\end{array}$

$3.1 \quad p$-variation of $\mathrm{fBm} \ldots \ldots \ldots \ldots \ldots$

3.2 Theorems Concerning Stieltjes Integration on $\mathrm{fBm} \ldots \ldots$

\begin{tabular}{|ll}
\hline & Itô Integral with Brownian Motion as the Integrator \\
\hline
\end{tabular}

$4.1 \quad$ Brownian Motion is Nowhere Differentiable almost surely $\ldots . . . .20$

4.1.1 The Impacts of Brownian Motion's nowhere-Differentiability on Itô Calculus when Brownian Motion is the Integrator $\ldots \quad$. . 20

4.2 Brownian Motion has Non-zero Quadratic Variation Almost Surely . 21

4.2.1 The Relationship between Quadratic Variation and Differen-

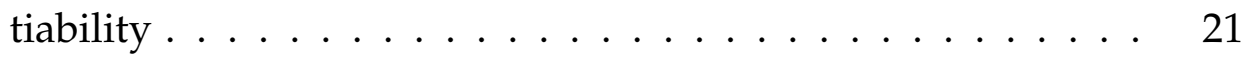

4.2.2 Quadratic Variation of Nowhere-differentiable Brownian Mo-

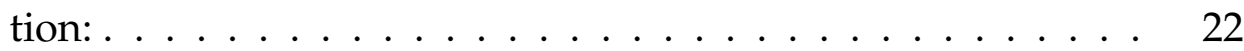

4.3 Brownian Motion is a Function of Unbounded Variation Almost surely 23

4.3 .1 Functions of Bounded Variation $\ldots \ldots \ldots \ldots$

4.3 .2 Bounded Variation and Bounded Functions . . . . . . . . . . 23

4.3.3 Bounded Variation and Monotonically Increasing Functions . 24

4.3 .4 Bounded Variation and Differentiable Functions . . . . . . . . 24

4.3.5 Brownian Motion has Unbounded Variation Almost Surely . 26

4.4 Brownian Motion in Contrast to a Smooth Function in Standard Cal-

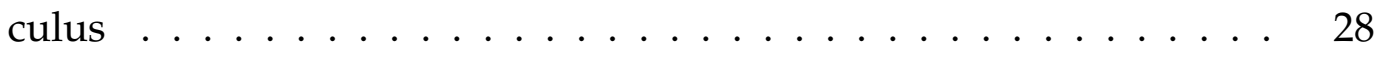

4.5 Itô Integral Defined for Random Step Process $\ldots \ldots \ldots \ldots \ldots$

4.5 .1 Definition . . . . . . . . . . . . . . . . . 28

4.5.2 Other Properties of Itô Integral Defined on Simple Processes . 29

4.5 .3 Itô Isometry $\ldots \ldots$

$4.6 \quad$ Itô Integral Defined for General $\mathcal{H}_{0}^{2}$ Integrands $\ldots \ldots \ldots$

4.6 .1 Construction of the general Itô Integral $\ldots \ldots \ldots$

4.6 .2 Summary of the Steps to Construct the Itô Integral . . . . . . . 32 
4.6 .3 Itô Isometry $\ldots \ldots \ldots$

4.7 Differences between Itô Integral and Riemann Integral $\ldots \ldots$

5 Itô-Doeblin Formula

5.1 Quadratic Variation . . . . . . . . . . . . . . . . . . . . 35

5.1 .1 Quadratic Variation of a Brownian Motion . . . . . . . . . . 35

5.1 .2 Quadratic Variation of an Itô Integral . . . . . . . . . . . . 35

5.1 .3 Quadratic Variation of a continuous Itô Process . . . . . . . . 37

5.1 .4 Quadratic Variation of a Jump Process . . . . . . . . . . . 37

5.2 Itô Formula for Brownian Motion . . . . . . . . . . . . . . . . 38

5.3 Itô Formula for continuous Itô Process $\ldots \ldots \ldots$

5.4 Itô Formula for Jump Process $\ldots \ldots \ldots \ldots \ldots$

5.5 Itô Formula for Multiple Jump Process $\ldots \ldots \ldots \ldots$

6 Stochastic Integral with Jump-Diffusion Processes as the Integrator $\quad 42$

6.1 Itô Integral with Continuous Itô Process as the Integrator $\ldots \ldots$

6.2 Stochastic Integral with Pure Jump Process as the Integrator $\ldots . . \quad 42$

6.3 Stochastic Integral with Jump-Diffusion Process as the Integrator . . 43

6.3.1 More on Itô-Doeblin Formula for Jump Stochastic Integral . . 43

6.4 S.D.E. with Jumps $\ldots \ldots \ldots \ldots$

\begin{tabular}{|lll}
\hline & Jump-Diffusion Processes in Financial Applications & 47
\end{tabular}

7.1 Volatility and Quadratic Variation of Diffusion Models $\ldots . . . . .47$

7.1 .1 Concepts of Volatility in Diffusion Models: . . . . . . . . . . . 47

7.2 Some Financial Instruments: . . . . . . . . . . . . . . . . . . . . . 48

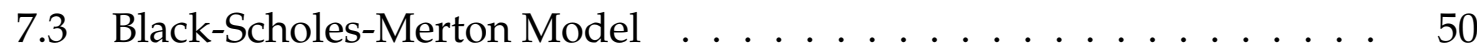

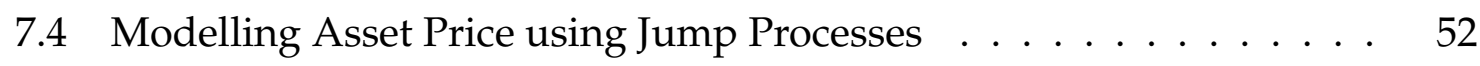

$7.4 .1 \quad$ Merton Jump-Diffusion Model $\ldots \ldots \ldots$. . . . . . . . . . 54 
8 Application in Monte Carlo Simulation $\quad 56$

8.1 Fractional Brownian Motion Paths . . . . . . . . . . . . . . 56

8.1.1 Euler Hypergeometric Integrals . . . . . . . . . . . . . 56

8.1 .2 Gaussian Quadrature . . . . . . . . . . . . . . 57

8.2 Simulation of Black-Scholes-Merton Model . . . . . . . . . . . . . . . 57

8.3 Simulation of Merton Jump Diffusion Model . . . . . . . . . . . . . . 60

8.3.1 Simulation at Fixed Dates . . . . . . . . . . . . . . 60

8.4 Empirical Examination of Daily Returns on Standard \& Poor 500 Index 63

\begin{tabular}{|l|l}
\hline Appendices & 67
\end{tabular}

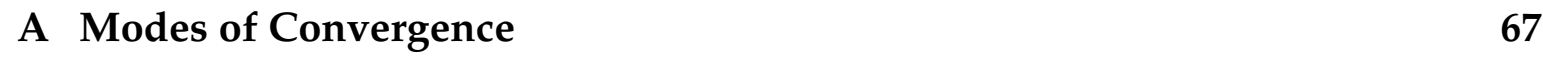

A.1 Mean Square Convergence . . . . . . . . . . . . . . . . . . . . . . . . 67

\begin{tabular}{lll}
\hline B & Function Approximation and Convergence in $\mathcal{L}^{2}$-Norm & 68
\end{tabular}

B.1 Sufficient Conditions for Convergence in $\mathcal{L}^{2} \ldots \ldots$. . . . . . . . . 68

B.2 Function Approximation in $\mathcal{L}^{2}$-Norm $\ldots \ldots . \ldots . \ldots 6$

C Function Approximation and Convergence in Euclidean Norm $\quad 69$

\begin{tabular}{|l|l|}
\hline & From Euclidean Convergence to $\mathcal{L}^{2}$ Convergence
\end{tabular}

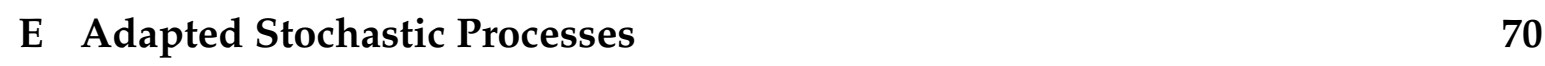

\begin{tabular}{ll}
\hline Bibliography & 71
\end{tabular}

\section{List of Figures}

$1 \quad$ Brownian Motions with almost surely nowhere-differentiable paths . 25

$2 \quad$ A Fractional Brownian Motion Path with $H=0.3$. . . . . . . . . . 58

$3 \quad$ A Fractional Brownian Motion Path with $H=0.7$. . . . . . . . . . . 58 
4 Simulation of a Black-Scholes-Merton Diffusion Process Path . . . . . 59

5 QQ Plot of a Black-Scholes-Merton Diffusion Process . . . . . . . . . 59

6 Simulation of a Merton Jump Diffusion Process using Fixed Dates Scheme . . . . . . . . . . . . . . . . . . . 62

$7 \quad$ QQ Plot of a Merton Jump Diffusion Process using Fixed Dates Scheme 62

$8 \quad$ Log Daily Return S\&P 500 Index: from 4 Jan 2000 to Aug 82013 . . . 63

9 Histogram of Log Return S\&P 500 Index Compared to Standard Normal 63 


\section{Introduction}

The objective of this thesis is to review the development of integrals for some classes of stochastic processes and their applications to finance. The reorganization of some literature results which will be included will appear in following order. First we will introduce those useful stochastic processes which will be showcased as Brownian motion, fractional Brownian motion in the Gaussian processes class, continuous Itô processes as well as jump-diffusion processes. And then in the next chapter, we will present the Stieltjes integral, including Young's extension to incorporate $p$-variation into the general Stieltjes theory. From there, we show that fractional Brownian motion (fBm) with Hurst parameter $H>\frac{1}{2}$, can also be compatible with the $p$-variation requirements on general Stieltjes integration. However, as soon as $H=\frac{1}{2}$, which gives us a standard Brownian motion, it makes insufficient conditions to properly define a Stieltjes integral and that is due to special properties of Brownian motion, which include but not limited to the equivlency of a finite $p>2$ variation, unbounded variation, nowhere differentiability and positive quadratic variation. Therefore, a new Itô integral is developed with Brownian motion as the integrator, and we introduce its calculus in Chapter 4. We single out a section on a summary of the quadratic variation properties of all the stochastic processes from Brownian motion to jump-diffusion processes and how they have a close relationship with the Itô-Doeblin formula as the "chain" rule in Itô calculus in Chapter 5. The Itô integral extension to continuous Itô processes, pure jump processes and further to jump-diffusion processes are shown in Chapter 6. Thereafter, applications in modelling financial assets are presented in Chapter 7 and an examination of emprical data of Standard \& Poor 500 index from 2000 January $4^{\text {th }}$ to 2013 August $8^{\text {th }}$ as well as my own outcome in simulations using MATLAB of the Black-ScholesMerton (BSM) model and the Merton jump-dffusion model (MJD) are done in Chapter 8 and we can clearly see an improvement from the BSM model to MJD model to 
incorporate the fat tail effects exhibited in the empirical data. 


\section{Useful Stochastic Processes}

\subsection{Gaussian Process}

Definition 1.1 A process $\left\{X_{t}\right\}_{t \in T}$ for some index set $T$ is called a Gaussian process if and only if for every finite set of indices $t_{1}, \ldots, t_{k} \in T,\left(X_{t_{1}}, \ldots, X_{t_{k}}\right)$ is a multivariate Gaussian random variable.

\subsection{Brownian Motion}

Definition 1.2 A stochastic process $B(t)$ is called a standard Brownian Motion or Wiener Process if it satisfies:

1. $B(t)$ starts from 0 , i.e. $B(0)=0$

2. B(t) has a continuous path

3. $B(t)$ has independent and stationary increments:

- $B(t)-B(s)$ and $B(v)-B(u)$ are independent when $s<t \leq u<v$

- $B(t)-B(s)={ }^{d} B(t+h)-B(s+h) \sim N(0, t-s)$ for $s, t \in[0, \infty), t \geq s$ and $h$ for $t+h, s+h \in[0, \infty)$

Brownian motion is a Gaussian process.

\subsection{Fractional Brownian Motion (fBm)}

Definition 1.3 A continuous time centered Gaussian process $B_{H}(t)$ for $t \in[0, T], H \in$ $(0,1)$ is a fractional Brownian motion when:

1. $B_{H}(0)=0$.

2. $E\left[B_{H}(t)\right]=0, \forall t \in[0, T]$.

3. $E\left[B_{H}(t) B_{H}(s)\right]=\frac{1}{2}\left(|t|^{2 H}+|s|^{2 H}-|t-s|^{2 H}\right)$. 


\subsubsection{Comparison with standard Brownian motion}

$\mathrm{fBm}$ is a generalization of Brownian motion not necessarily with independent increments. It is a Brownian motion when $H=\frac{1}{2}$.

\subsubsection{Properties in terms of $\mathrm{H}$ :}

- $H=\frac{1}{2}$ : Brownian motion.

- $H>\frac{1}{2}$ : Positively correlated increments, also a persistent $\mathrm{fBm}$.

- $H<\frac{1}{2}$ : Negatively coorelated increments, also an anti-persistent fBm.

\subsubsection{Other Properties:}

We provide more properties of $\mathrm{fBm}$ as discussed in Biagini, Hu, Øksendal and Zhang (2008)

1. $\mathrm{fBm}$ has stationary increments, which means $B_{H}(s+t)-B_{H}(s)={ }^{d} B_{H}(t)$ for $s, t \geq 0$,

2. $\mathrm{fBm}$ has continuous sample paths.

\subsubsection{Applications:}

$\mathrm{fBm}$ is applied in the modeling of many situations such as introduced in Biagini, Hu, Øksendal, Zhang (2008):

1. The temperature at a specific place as a function of time.

2. The level of water in a river as a function of time.

3. The values of the log returns of a stock.

4. Financial turbulence, i.e. the empirical volatility of a stock, and other turbulence phenomena 
5. The prices of electricity in a liberated electricity market.

In cases 1 to 3 , we use persistent $\mathrm{fBm}$ with $H>\frac{1}{2}$ and cases 4 and 5 anti-persistent with $H<\frac{1}{2}$ where persistent means that the particle persist in the same direction and anti-persistent means that the particle path tends to turn back on itself as described in Sarkar (2011).

\subsection{Itô Process}

Almost all Markov processes other than jump processes belong to the class of Itô processes.

Definition 1.4 An Itô process $X(t)$ can be expressed in the form of

$$
X(t)=X(0)+\int_{0}^{t} \Delta(u) d B(u)+\int_{0}^{t} \Theta(u) d u
$$

where $B(u)$ is a Brownian motion with $\{\mathcal{F}(u)\}_{u \geq 0}$ being the associated filtration; $\Delta(u)$ and $\Theta(u)$ are the adapted processes. 11

In (1), $\int_{0}^{t} \Delta(u) d B(u)$ is the Itô integral part, denoted by $I(t)$, $\int_{0}^{t} \Theta(u) d u$ is the Riemann integral part, denoted as $R(t)$.

The detailed description will be given in Sections 4-6.

\subsection{Jump Processes}

\subsubsection{Pure Jump Process}

- the Building Block of Jump Stochastic Integrals

A typical feature of a pure jump processes is that they stay constant between jumps. Types:

- Simple Poisson Process

\footnotetext{
${ }^{1}$ See Appendix E
} 
- Compound Poisson Process

\section{Simple and Compound Poisson Process:}

Let $N(t)$ be the simple Poisson. We can then define the Compound Poisson process $Q(t) b y$

$\mathrm{Q}(\mathrm{t})=\mathrm{Q}(0)+\sum_{k=1}^{N_{t}}\left[Y_{T_{k}}-Y_{T_{k}-}\right]$, where $T_{k}$ are the jump times and $Y\left(T_{k}\right)$ are i.i.d. random variables.

Note that in the special case in which $Y_{T_{k}}-Y_{T_{k^{-}}}=1$ for all $\mathrm{k} Q(t)=N(t)$. We then have the properties

- right continuous: $N(t+)=N(t)$

- piece-wise constant path: $Q\left(T_{k+1}-\right)-Q\left(T_{k}\right)=0$

- random jump sizes.

- Lévy properties: Independent and Stationary Increments:

$-N(s+t)-N(s)={ }^{d} N(t):$

The distribution of the number of increments only depends on the length of the time interval, but not on the start and end time

- $N(t+s)-N(s)$ is independent of $N(s)$ : The increments from the present is independent from the past up to the present.

\section{Properties:}

1. A pure jump process has a stochastic integral representation w.r.t. a pure jump process.

2. Because the simple Poisson has Lévy properties, so does the Compound Poisson, that is:

- $Q(t+s)-Q(s)={ }^{d} Q(t)$ 
- $Q(t+s)-Q(s)$ is independent of $Q(s)$.

\section{Construction:}

$Q(t)=\sum_{i=1}^{N_{t}} Y_{i}$

$Y_{i}$ : i.i.d. random variable with probability distribution $v(d y)$ on $R$

$N(t)$ : Simple Poisson Process; $Q(t)$ share the same jump times as $N(t)$

$d Q(t)=Q(t)-Q(t-)=Y_{i}$ when $t \in\left\{T_{1}, \ldots, T_{N(t)}\right\}$, where $T_{k}$ are the jump times and 0 otherwise.

\section{Expression of the Compound Poisson Process as a Jump Stochastic Integral:}

1. Simple $N(t)$ : from a sum of jump sizes 1 to a Stochastic Integral

$N(t)=\sum_{k=1}^{N_{t}} I_{\left[T_{k}, \infty\right)}(t) ; T_{k}$ is the jump times for $k=1, \ldots N(t)$

$=\int_{0}^{t} 1 d N_{t}=\int_{0}^{t}$ jump sizes $d N_{t}=\int_{0}^{t}(N(s)-N(s-)) d N(s)$.

2. Compound $Q(t)$ : from a sum of random jump sizes to a Stochastic Integral $Q(t)=\sum_{k=1}^{N_{t}} Y_{i} ; Y_{i}$ is the random jump sizes and independent of $N(t)$ $Q(t)=\int_{0}^{t} Q(s)-Q(s-) d N(s)=\int_{0}^{t}$ jump sizes $d N(s)=\int_{0}^{t} d Q(t)$.

\section{Jump Stochastic Integration Produces Jump Process:}

1. The stochastic integration w.r.t. a simple Poisson process produces a Compound Poisson process: $\int_{0}^{t} \phi(s) d N(s)=\sum_{k=1}^{N(t)} \phi\left(T_{k}\right)$; where $\phi(s)$ is a stochastic process.

2. The stochastic integration w.r.t. a compound Poisson process produces again a compound Poisson process:

$\int_{0}^{t} \phi(s) d Q(s)=\sum_{k=1}^{N_{t}} \phi\left(T_{k}\right)\left(Q\left(T_{k}\right)-Q\left(T_{k}-\right)\right)$; where $T_{k}$ are the jump times of the Compound Poisson $Q(t)$ and $\left(Q\left(T_{k}\right)-Q\left(T_{k}-\right)\right)$ are the jump sizes of $Q(t)$. 


\subsubsection{General Jump Process}

Definition 1.5 A general jump process $X(t)$ can be written in the form of $([21])$

$$
X(t)=X(0)+R(t)+I(t)+J(t)
$$

where $R(t)$ is a Riemann integral $\int_{0}^{t} \Theta(u) d u$ with $\Theta(u)$ being an adapted process, $I(t)$ is an Itô integral $\int_{0}^{t} \Gamma(u) d B(u)$ with $\Gamma(u)$ being an adapted process and the integrator is a Brownian motion $B(t)$ and $J(t)$ is an adapted, right-continuous pure jump process and $J(0)=0$.

We denote the continuous part $X(0)+R(t)+I(t)$ as $X^{c}(t)$, and we permit only finitely many jumps over a finite time interval for $J(t)$.

\section{Properties:}

1. A general jump process $X(t)$ is right-continuous, which means $\lim _{s \downarrow t} X(s)=$ $X(t), X(t)$ is the value after the jump while $X(t-)=X(0)+R(t)+I(t)+$ $J(t-)$ is the value before the jump. Hence, the jump size $\Delta X(t)=X(t)-$ $X(t-)=\Delta J(t)=J(t)-J(t-)$.

2. Any jump process can be expressed as a stochastic integral with jump process as the integrator.

3. A jump process is an Itô process. 


\section{Introduction to Stieltjes Integral}

\subsection{Definitions and Notations}

- $f(x)$ is defined on a closed interval $[a, b]$;

- $\alpha$ is a function not necessarily continuous;

- $\alpha(x)$ is a monotonic increasing function on $[a, b]$;

- $[a, b]$ is divided into partitioned intervals: $\left[x_{i}, x_{i+1}\right) ; x_{0}=a ; x_{n}=b$

- $M_{i}$ : the lub of $\mathrm{f}(\mathrm{x})$ in $\left[x_{i}, x_{i+1}\right) ; m_{i}$ : the glb of $\mathrm{f}(\mathrm{x})$ in $\left[x_{i}, x_{i+1}\right)$

- $\Delta \alpha_{i}=\alpha\left(x_{i+1}\right)-\alpha\left(x_{i}\right)$

- $P_{n}$ denotes a partition of $[a, b]$ such that $P_{n}=\left\{a=x_{1}<x_{2}<\ldots<x_{n}=b\right\}$

- Mesh $\Pi\left(P_{n}\right)$ is the maximum of the size of the partition sub-intervals such that $\Pi\left(P_{n}\right)=\max _{i}\left|x_{i+1}-x_{i}\right|$

- Upper Stieltjes Sum is defined as $U\left(P_{n}, f, \alpha\right)=\sum_{i=0}^{n-1} M_{i} \Delta \alpha_{i}$

- Lower Stieltjes Sum is defined as $L\left(P_{n}, f, \alpha\right)=\sum_{i=0}^{n-1} m_{i} \Delta \alpha_{i}$

- Upper Stieltjes Integral is defined as $\bar{\int}_{a}^{b} f d \alpha=g l b_{P_{n}} U\left(P_{n}, f, \alpha\right)$

- Lower Stieltjes Integral is defined as $\int_{a}^{b} f d \alpha=l u b_{P_{n}} L\left(P_{n}, f, \alpha\right)$

The introduction of following basic Stieltjes integral theorems can be seen in Rudin's (1964 book. 


\subsection{Theorems on Upper and Lower Stieltjes Sums}

Theorem 2.1 The sequence of Upper Stieltjes Sums is a monotonic decreasing sequence in $P_{n}$, i.e.,

$$
P_{n} \subset P_{m}^{*} \Rightarrow U\left(P_{n}, f, \alpha\right) \geq U\left(P_{m}^{*}, f, \alpha\right) .
$$

Similarly,

Theorem 2.2 The sequence of Lower Stieltjes Sums is a monotonic increasing sequence in $P_{n}$, i.e.,

$$
P_{n} \subset P_{m}^{*} \Rightarrow L\left(P_{n}, f, \alpha\right) \leq L\left(P_{m}^{*}, f, \alpha\right) .
$$

Theorem 2.3 The sequence of Upper Stieltjes Sums converges, and the limit is defined to be the Upper Stieltjes Integral

$$
U\left(P_{n}, f, \alpha\right) \longrightarrow \int_{a}^{b} f d \alpha
$$

Similarly,

Theorem 2.4 The sequence of Lower Stieltjes Sums converges, and the limit is defined to be the Lower Stieltjes Integral

$$
L\left(P_{n}, f, \alpha\right) \longrightarrow{\underline{\int_{a}^{b}}}_{a}^{b} d \alpha .
$$

Theorem 2.5 Upper Stieltjes Sum $\geq$ Lower Stieltjes Sum, in any Partition i.e. for any partitions $P_{i}, P_{j}$

$$
U\left(P_{i}, f, \alpha\right) \geq L\left(P_{j}, f, \alpha\right) .
$$

Theorem 2.6 Upper Stieltjes Integral $\geq$ Lower Stieltjes Integral i.e.

$$
\bar{\int}_{a}^{b} f d \alpha=g l b_{n} U\left(P_{n}, f, \alpha\right) ; \int_{a}^{b} f d \alpha=\operatorname{lu} b_{n} L\left(P_{n}, f, \alpha\right)
$$


and,

$$
\int_{a}^{b} f d \alpha \geq \int_{a}^{b} f d \alpha
$$

\subsection{Properties of Stieltjes Integrable Function}

Given an integrator $\alpha$ let $R(\alpha)$ denote the set of integrable functions.

Theorem 2.7 The space of Stieltjes Integrable functions $R(\alpha)$ is a linear vector space i.e. for $f(x), g(x)$ defined on $[a, b]$ and $f(x), g(x) \in R(\alpha), a, b$ are constants, then

$$
a f(x)+b g(x) \in R(\alpha)
$$

Theorem 2.8 $R(\alpha)$ is closed under continuous composite functions

i.e. for $f(x)$ defined on $[a, b]$ and $f(x) \in R(\alpha), m \leq f(x) \leq M, \phi(y)$ is a continuous function defined on closed and bounded interval $[m, M]$, then $\phi(f(x)) \in R(\alpha)$.

Theorem 2.9 From $R\left(\alpha_{1}\right)$ to $R\left(c \alpha_{1}+d \alpha_{2}\right)$

$f(x) \in R\left(c \alpha_{1}+d \alpha_{2}\right)$ if:

$f(x) \in R\left(\alpha_{1}\right)$ and $f(x) \in R\left(\alpha_{2}\right)$ and $c, d$ are constants. and

$$
\int_{a}^{b} f d\left(c \alpha_{1}+d \alpha_{2}\right)=c \int_{a}^{b} f d \alpha_{1}+d \int_{a}^{b} f d \alpha_{2}
$$

\subsection{Sufficient Conditions to Determine Stieltjes Integrability}

To define the basic Stieltjes integral as introduced in Rudin

$$
\int_{0}^{T} f(x) d \alpha(x)
$$

we make the assumptions on the integrator $\alpha(x)$ : 
- $\alpha(x)$ is not necessarily continuous

- $\alpha(x)$ is monotonically increasing on the compact support $[a, b]$.

We will show later that the above is equivalent to assuming bounded variation in the language of $p-$ variation.

To ensure the existence of the integral in (3) as a well-defined Stieltjes integral, we derive further the following requirements on the integrand function $f(x)$ and integrator $\alpha(x)$ to be Stieltjes integrable as introduced in Rudin's (1964) book.

\section{Definition of Stieltjes Integrability:}

Definition 2.1 For $f(x)$ on a closed and bounded interval $[a, b], f(x)$ is Stieltjes Integrable iff

$$
\bar{\int}_{a}^{b} f d \alpha=\int_{a}^{b} f d \alpha
$$

i.e.

$$
g l b_{n} U\left(P_{n}, f, \alpha\right)=\operatorname{lu} b_{n} L\left(P_{n}, f, \alpha\right)
$$

and we denote the Stieltjes Integral as

$$
\bar{\int}_{a}^{b} f d \alpha=\int_{a}^{b} f d \alpha=\int_{a}^{b} f d \alpha=g l b_{n} U\left(P_{n}, f, \alpha\right)=\operatorname{lub} b_{n} L\left(P_{n}, f, \alpha\right) .
$$

\subsubsection{Theorems to Determine Stieltjes Integrability}

\section{Definition 2.2}

For $f(x)$ on a closed and bounded interval $[a, b], f(x)$ is Stieltjes Integrable iff for $\forall \varepsilon>$ $0, \exists$ partition $P_{n}$ s.t.

$$
U\left(P_{n}, f, \alpha\right)-L\left(P_{n}, f, \alpha\right)<\varepsilon
$$

Theorem 2.10 For a continuous integrand functions on closed and bounded intervals the Stieltjes integral exists (Rudin (1964)), that is 
for $f(x)$ on a closed and bounded interval $[a, b], f(x)$ is Stieltjes Integrable if $f(x)$ is continuous.

PROOF. Recall that if we want to prove that $f(x)$ is Stieltjes integrable, we are to prove the Upper Stieltjes Sum and the Lower Stieltjes Sum coverge to the same limit, in other words, if we denote:

$U\left(P_{n}, f, \alpha\right)$ the Upper Stieltjes Sum and $L\left(P_{n}, f, \alpha\right)$ the Lower Stieltjes Sum $\Pi\left(P_{n}\right)$ the mesh of any partition $P_{n}$, which is $\max \Delta x_{i}$, for $i=1, \ldots, n$, then $\forall \epsilon>0, \exists \delta>0$ we need

$$
\Pi\left(P_{n}\right)<\delta \Rightarrow U\left(P_{n}, f, \alpha\right)-L\left(P_{n}, f, \alpha\right)<\epsilon
$$

Since $f(x)$ is a continuous function on the compact interval $[a, b], f(x)$ is uniformly continuous as a result, which means, for any $\eta>0, \exists \delta(\eta)>0$ such that we can make $|f(x)-f(t)|<\eta$ as long as $|x-t|<\delta$ for any $x, t \in[a, b]$, therefore:

$$
|x-t|<\delta(\eta) \Rightarrow M_{i}-m_{i}<\eta \Rightarrow U\left(P_{n}, f, \alpha\right)-L\left(P_{n}, f, \alpha\right)<\eta \sum_{i=1}^{n} \Delta \alpha_{i}
$$

And we can choose an arbitrary $\eta$ such that $\eta \sum_{i=1}^{n} \Delta \alpha_{i}<\epsilon$.

\section{Theorem 2.11}

For $f(x)$ on a closed and bounded interval $[a, b], f(x)$ is Stieltjes Integrable if $f(x)$ is monotonic on $[a, b]$ and $\alpha(x)$ is continuous on $[a, b]$, assuming $\alpha(x)$ is already monotonically increasing. 


\subsection{The General Stieltjes Integral Theory}

\subsubsection{Sufficient Conditions of the Existence of a Stieltjes Integral}

We say, in general, the Stieltjes integrability of an integrand function $f(t)$ depends on its properties of continuity and $p$-variation as well as the integrator $\alpha(t)^{\prime} \mathrm{s}$. As introduced in Kozdron's (2007) and Taylor's (1972), the $p$-variation, $V_{p}\left(f, a, b, P_{n}\right)$, of a real-valued function $f(t)$ defined on a closed interval $[a, b]$ and partitions of the form $P_{n}=\left\{0, t_{1}, \ldots, t_{N}=b\right\}$ with $\Pi\left(P_{n}\right)=\max \left(t_{i+1}-t_{i}\right)$ is defined as follows.

Definition 2.3 The true $p$ - th Variation of $f(t)$ on closed interval $[a, b]$ is

$$
V_{p}(f, a, b)=\sup _{P_{n}} Q_{p}
$$

where the supremum is taken over all partitions $P_{n}$ of $[a, b]$. Given an increasing sequence of partitions $P_{n}=\left\{0=t_{0}, t_{1}, \ldots, t_{n}=b\right\}$, we let

$$
Q_{p}\left(f, a, b, P_{n}\right)=\sum_{i=1}^{n}\left|f\left(t_{i}\right)-f\left(t_{i-1}\right)\right|^{p} .
$$

Note that we will later in Section 4.2 define the quadratic variation of a stochastic process $X, Q V(X, a, b)$, as the limit in $\mathcal{H}_{0}^{2}$ of $Q_{2}$, more specifically, it means

$$
Q V(X, a, b)=\lim _{\Pi\left(P_{n}\right) \rightarrow 0} Q_{2}\left(X, a, b, P_{n}\right)
$$

For an integrand function $f(u)$ and an integrator function $\alpha(u)$, the relationship between their properties and Stieltjes Integrability as described in Thomas Mikosch (1998) and L.C. Young (1936), is shown in the following tree: 


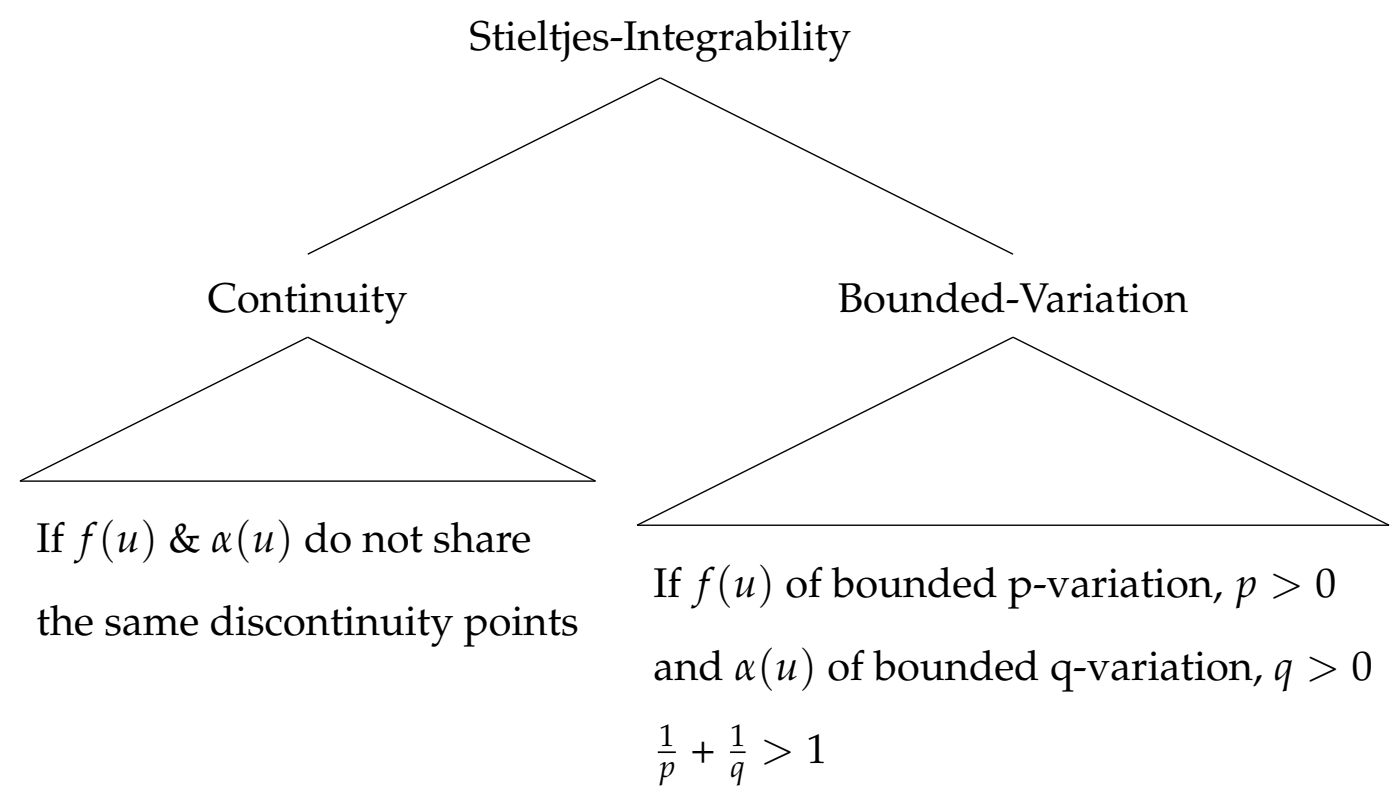

In particular, we describe 2 types of integrator function in this section, one has bounded variation that makes Stieltjes integral well-defined and Brownian motion with unbounded variation that makes Stieltjes integral unemployable.

\section{When the integrator $\alpha(x)$ is of bounded variation, finite $p=1$ variation}

We can see that when $p=1$, in order to satisfy the condition $\frac{1}{p}+\frac{1}{q}>1$, we need $\frac{1}{q}>0$, this is true for any $q>0$. Hence, as long as one of the integrand or integrator has bounded variation, the Stieltjes integral can be well-defined with respect to the $p$-variation requirements.

The situation of bounded variation is equivalent to setting it as a difference of 2 monotonically increasing functions as the following theorems on sufficient conditions of a bounded variation function say in the introduction of Feldman's (2008):

Theorem 2.12 A function $\alpha(x):[a, b] \rightarrow \mathcal{R}$ has bounded variation if and only if it is a difference of two monotonically increasing functions.

Theorem 2.13 If $\alpha(x):[a, b] \rightarrow \mathcal{R}$ is monotonically increasing, then $\alpha(x)$ has bounded variation and $V_{1}(\alpha, a, b)=\alpha(b)-\alpha(a)$. 
What's more, we can also recall, Stieltjes integral has linearity property on the integrator function in theorem 2.9. $f(x) \in R\left(\alpha_{1}\right)$ and $f(x) \in R\left(\alpha_{2}\right) \Rightarrow f(x) \in R\left(c \alpha_{1}+\right.$ $\left.d \alpha_{2}\right)$ and this is because in $p$-variation theory: Feldman's (2008):

Theorem 2.14 If $\alpha_{1}(x), \alpha_{2}(x):[a, b] \rightarrow \mathcal{R}$ have bounded variation and $c, d \in \mathcal{R}$, then $c \alpha_{1}+d \alpha_{2}$ has bounded variation too.

\section{Sufficient conditions of Stieltjes Integrability}

When $\alpha(x)$ is of bounded variation:

$$
\text { Stieltjes exists } \begin{cases}\text { No common discontinuities } & \text { if } f(x) \text { is continuous } \\ \frac{1}{p}+\frac{1}{q}>1 & \text { or if } \alpha(x) \text { is continuous }\end{cases}
$$

If we recall our earlier definition on Stieltjes integral as in Rudin (1964), we set the integrator function $\alpha(x)$ as monotonically increasing, hence it is of bounded variation and we proved in Theorem 2.10, the Stieltjes integral is well-defined for continuous integrand $f(x)$ or continuous integrator $\alpha(x)$ and there is no restriction on the finite $q$-variation of $f(x)$ according to the formula $\frac{1}{p}+\frac{1}{q}>1$.

\section{When the integrator is of unbounded variation, finite $p>1$ variation}

For our coming purposes, we are interested in the $p$-variation of Brownian motion, in particular, when $p>2$ and the role they play in the theory of integration. Firstly, from Kozdron's (2007) and Taylor's (1972), we have the following theorems in terms of the $p$-variation for the Brownian motion:

Theorem 2.15 Brownian motion has finite true $p$ - variation if and only if $p>2$ :

$$
V_{p}(B(t), a, b)<\infty \Leftrightarrow p>2
$$

We will also later show that: 
Theorem 2.16 $Q V(B(t), a, b)=(b-a)$, almost surely.

We will provide more details on these properties as well as others of Brownian motion in the next chapter associated to Itô integral using Brownian motion as an integrator. So, when we are interested in an integral with $B(u)$ of a Brownian motion path as the integrator,the analogue of the previous tree is:

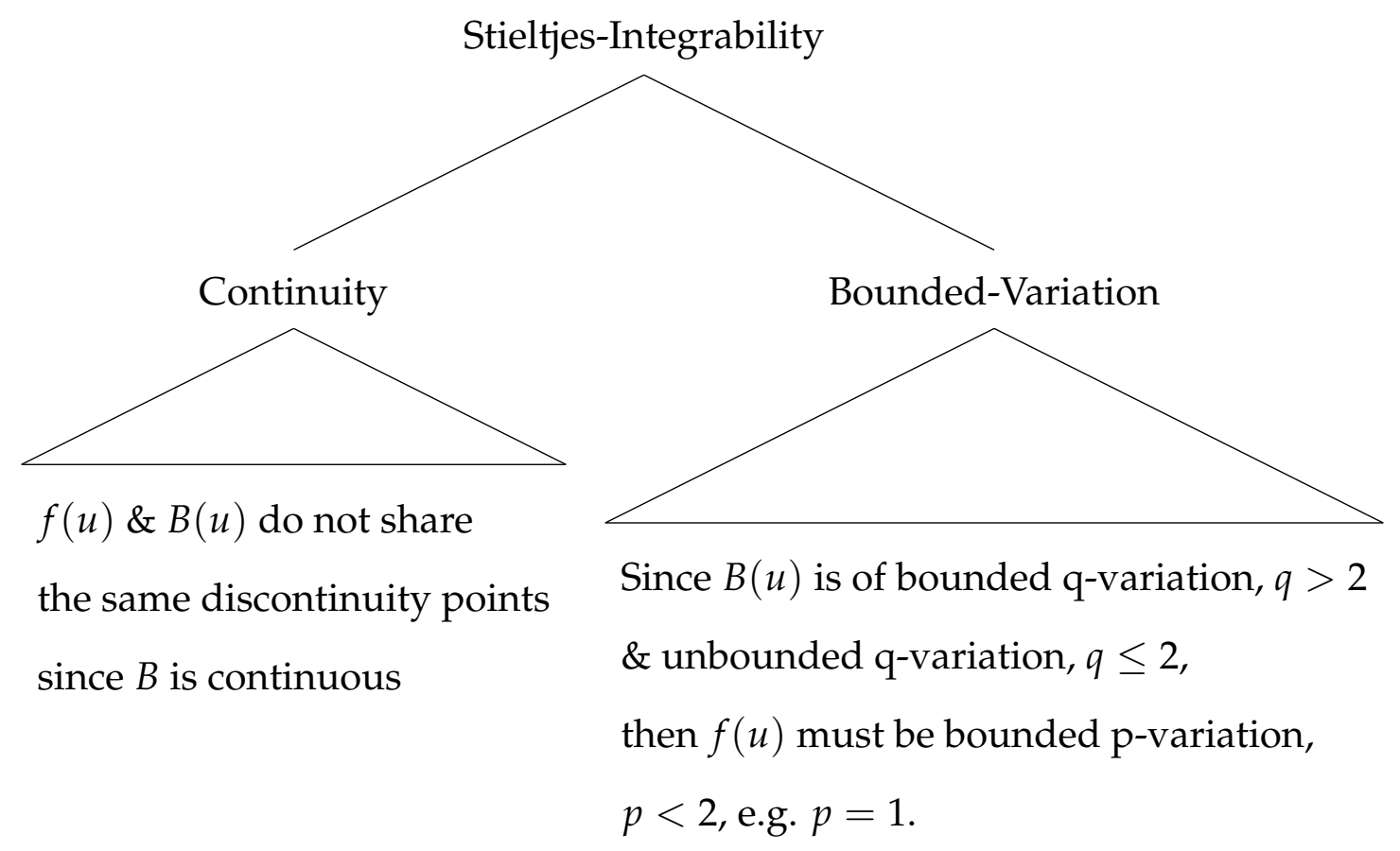

Hence, when the integrator $B(u)$ is of unbounded q-variation when $q \leq 2$ as the Brownian motion path, the integrand function $f(u)$ has to be of bounded p-variation for $p<2$ for the Stieltjes Integral to be defined, in other words, $\int_{0}^{t} B(u) d B(u)$ is not defined as a Stieltjes Integral.

This motivates a further discussion on integrals associated with Brownian motion as the integrator, please refer to Chapter 4 -Ito Integral with Brownian Motion as the Integrator. But first let's see that Stieltjes integral $\int_{0}^{t} B_{H}(u) d B_{H}(u)$ can still be defined on a special generalization of the Brownian motion - with the integrator being a fractional Brownian motion $(\mathrm{fBm}) B_{H}(u)$. 


\section{Stieltjes Integral with Fractional Brownian Motion as}

\section{the Integrator}

For a recap, as we mentioned earlier, in Young's extension on general Stieltjes theory, $\frac{1}{p}+\frac{1}{q}>1$ is the condition that allows the Stieltjes integral $\int_{0}^{T} f(t) d g(t)$ well-defined when $f(t)$ is of finite p-variation and $g(t)$ with finite q-variation. Clearly when $g(t)$ is the standard Brownian motion $B(t), q>2$, then $p$ needs to be smaller than 2 , in other words, $p<2$ for the integral to exist. Hence, the integral $\int_{0}^{T} B(t) d B(t)$ is not defined in the Stieltjes sense. However, we say that the integral $\int_{0}^{T} B_{H}(t) d B_{H}(t)$ can be well-defined in the Stieltjes sense when $B_{H}(t)$ is a fractional Brownian motion with $H \neq \frac{1}{2}$ because it has different finite $\mathrm{p}$-variation properties from a standard Brownian motion. A standard Brownian motion is a special case of a fractional Brownian motion when $H=\frac{1}{2}$. We will provide more details in the following, which are explained well in Salopek's (1998).

\section{$3.1 p$-variation of $\mathbf{f B m}$}

In particular, the following theorems are true about the finite $p$-variation of the $\mathrm{fBm}$ as mentioned in Salopek (1998) and Kawada and Kôno (1973):

Theorem 3.1 fBm has finite $p$-variation almost surely if and only if $p>\frac{1}{H}$.

Note that this theorem, Theorem 3.1 about $p$-variation of $\mathrm{fBm}$ in the general case is compatible with the special case when $H=\frac{1}{2}$, which gives a standard Brownian motion, Theorem 2.15 requiring $p>2$.

- Case 1: $\frac{1}{2}<H<1, \Rightarrow 1<\frac{1}{H}<2$

- Case 2: $\frac{1}{2}>H>0, \Rightarrow 2<\frac{1}{H}$

The second case above coincides with the case with standard Brownian motion as the integrator, therefore we are putting more attention on the 1st case here. We see 
that in Case 1, with $2>\frac{1}{H}>1$, it gives us the possibility for the integrator $\mathrm{fBm}$ $B_{H}(t)$ to have a finite $\mathrm{p}$-variation with $1<p<2$, hence $\int_{0}^{T} B_{H}(t) d B_{H}(t)$ is possible to be defined as a Stieltjes integral since $\frac{1}{p}+\frac{1}{p}>1$ when $p<2$ and we have a series of results in this situation as explained in Salopek (1998), which are shown in the following section.

\subsection{Theorems Concerning Stieltjes Integration on $\mathrm{fBm}$}

Lemma 3.1 Let $f(t)$ be a continuous function with finite p-variation with $1 \leq p<2$. Then for any $r \geq p$ such that $\frac{1}{p}+\frac{1}{q}>1$,

$$
E_{a}(f(t))=\exp \{f(t)-f(a)\}, t \in[a, b]
$$

is the unique solution as a finite r-variation funtion of the integral equation

$$
F(y)=1+\int_{0}^{y} F(u) d f(u), y \in[a, b] .
$$

Theorem 3.2 The case $\frac{1}{2}<H<1$, geometric fractional Brownian motion $P_{H}=\left\{P_{H}(t)\right.$ : $t \geq 0\}$ gives a unique solution of the integral equation

$$
F(t)=1+\int_{0}^{t} F(s) d\left(\mu s+\sigma B_{H}(s)\right), t \in[0, T]
$$

where $B_{H}(s)$ is of finite p-variation and from $\frac{1}{2}<H<1,1<p<2$ and $P_{H}=\left\{P_{H}(t):=\right.$ $\left.\exp \left(\mu t+\sigma B_{H}(t)\right)\right\}$. 


\section{Itô Integral with Brownian Motion as the Integrator}

\subsection{Brownian Motion is Nowhere Differentiable almost surely}

If we were to say that Brownian Motion $B(t)$ is differentiable almost surely at some time $t$, then it would mean:

$$
\lim _{h \rightarrow 0} \frac{B(t+h)-B(t)}{h}=B^{\prime}(t) \text { a.s. }
$$

for $B^{\prime}(t)$ being a finite-value random variable.

However, for a fixed time $t$, we all know that $B(t+h)-B(t) \sim \sqrt{h} N(0,1)$ and $\frac{B(t+h)-B(t)}{h} \sim \frac{1}{\sqrt{h}} N(0,1)$ and $\frac{1}{\sqrt{h}} N(0,1)$ does not converge in distribution when $h$ goes to 0 .

More specifically, for Brownian motion to be nowhere differentiable almost surely over $t \in[0, T]$, it means:

$$
P\left\{\bigcup_{t \in[0, T]} A_{t}\right\}=0
$$

where the event $A_{t}:=\left\{\lim _{h \rightarrow 0} \frac{B(t+h)-B(t)}{h}\right.$ exists $\}$. This was proved by Paley, Wiener and Zygmund (1933). A reference can be seen in Karatzas and Shreve's (1988).

\subsubsection{The Impacts of Brownian Motion's nowhere-Differentiability on Itô Cal- culus when Brownian Motion is the Integrator}

\section{Itô Integral using Brownian Motion as the Integrator}

The Itô integral $\int_{0}^{t} f(u) d B(u)$, which will be defined later is different from the ordinary Stieltjes integral $\int_{0}^{t} f(u) d g(u)$ when $g(u)$ is a differentiable function, where we can write $d g(u)$ as $g^{\prime}(u) d u$.

\section{Interpretation of Brownian Motion's nowhere Differentiability}

To get an interpretation about the differentiability of a function, we can view this to 
mean that when a function has a non-zero derivative at a point, then the function is monotonic on an interval around that point.

If we are to interpret the non-differentiability of a Brownian motion path, it gives us the intuition that the change in $d B(t)$ is much bigger than $d t$ within the time interval when $d t$ goes to 0 as explained in Visser's (2009).

\subsection{Brownian Motion has Non-zero Quadratic Variation Almost Surely}

\subsubsection{The Relationship between Quadratic Variation and Differentiability}

Given a set of partitions on the time interval $P_{n}=\left\{0=t_{0}, t_{1}, \ldots, t_{n}=T\right\}$, for the function $f(t)$ with first order derivative $f^{\prime}(t)$ defined everywhere and continuous, the Quadratic Variation is defined as follows, as introduced in Shreve's (2004):

\section{Definition 4.1}

$$
[f, f](T)=\lim _{\Pi\left(P_{n}\right) \rightarrow 0} \sum_{j=0}^{n-1}\left[f\left(t_{j+1}\right)-f\left(t_{j}\right)\right]^{2} .
$$

\section{Functions of Bounded Derivatives Has 0 Quadratic Variation}

Theorem 4.1 If the function $f(t)$ has derivative $f^{\prime}(t)$ defined everywhere and bounded, then

$$
[f, f](T)=0
$$

In particular if the derivative $f^{\prime}(t)$ is continuous, then it means that $\int_{0}^{T} f^{\prime}(t)^{2} d t$ is finite and $f(t)$ has 0 quadratic variation.

If $f^{\prime}(t)$ is not continuous and $\int_{0}^{T}\left|f^{\prime}(t)\right|^{2} d t$ is not guaranteed finite, then $[f, f](T)$ can be anywhere between 0 and $\infty$ 


\subsubsection{Quadratic Variation of Nowhere-differentiable Brownian Motion:}

Lemma 4.1 Consider a Brownian motion $B(\omega, t)$ when $t \in[0, T]$ and

$Q_{n}(T)=\sum_{j=1}^{n}\left|B\left(t_{j}\right)-B\left(t_{j-1}\right)\right|^{2}$ over a partition $v_{n}=\left\{0=t_{0}, \ldots, t_{n}=T\right\}$, then $Q_{n}(T)$ converges to $T$ in mean square.

ProOF. $\quad Q_{n}(T)$ converges to $T$ in mean square if $\lim _{n \rightarrow \infty} E\left[\left|Q_{n}(T)-T\right|^{2}\right]=0$. Since $E\left[Q_{n}(T)\right]=T, E\left[\left|Q_{n}(T)-T\right|^{2}\right]=\operatorname{Var}\left[Q_{n}(T)\right]=\operatorname{Var}\left[\sum_{j=1}^{n}\left|B\left(t_{j}\right)-B\left(t_{j-1}\right)\right|^{2}\right]$. By the independent and stationary increments of Brownian motion $B(\omega, t), \operatorname{Var}\left[Q_{n}(T)\right]=$ $\sum_{j=1}^{n} \operatorname{Var}\left|B\left(t_{j}\right)-B\left(t_{j-1}\right)\right|^{2}$. For a normal distribution $N\left(0, t_{j}-t_{j-1}\right)$, because the standard normal distribution has the fourth moment equal 3 and the second moment equal 1,

$$
\begin{aligned}
\operatorname{Var}\left[N\left(0, t_{j}-t_{j-1}\right)^{2}\right] & =E\left[\left(t_{j}-t_{j-1}\right)^{2} N(0,1)^{4}\right]-\left\{E\left[\left(t_{j}-t_{j-1}\right) N(0,1)^{2}\right]\right\}^{2} \\
& =\left(t_{j}-t_{j-1}\right)^{2}(3-1) \\
& =2\left(t_{j}-t_{j-1}\right)^{2}
\end{aligned}
$$

Hence,

$$
\begin{aligned}
\operatorname{Var}\left[Q_{n}(T)\right] & =2 \sum_{j=1}^{n}\left(t_{j}-t_{j-1}\right)^{2} \\
& \leq 2 \max _{j}\left(t_{j}-t_{j-1}\right) \sum_{j=1}^{n}\left(t_{j}-t_{j-1}\right) \\
& =2 T \max _{j}\left(t_{j}-t_{j-1}\right)
\end{aligned}
$$

and this goes to 0 following $\max _{j}\left|t_{j}-t_{j-1}\right|$ goes to 0 when $\operatorname{mesh}\left(v_{n}\right)$ goes to 0 as $n \rightarrow \infty$.

Theorem 4.2 For almost surely nowhere differentiable Brownian Motion B $(t)$, the Quadratic Variation $[B, B](T)=T$ almost surely. 
Proof. Consider a Brownian motion $B(\omega, t)$ when $t \in[0, T]$ and $Q_{n}(T)=\sum_{j=1}^{n}\left|B\left(t_{j}\right)-B\left(t_{j-1}\right)\right|^{2}$ over a partition $v_{n}=\left\{0=t_{0}, \ldots, t_{n}=T\right\}$, since $Q_{n}(T)$ converges to $\mathrm{T}$ in mean square, $Q_{n}(T)$ converges to $\mathrm{T}$ in probability, and therefore converges to $\mathrm{T}$ almost surely on a subsequence. 2

\subsection{Brownian Motion is a Function of Unbounded Variation Al- most surely}

\subsubsection{Functions of Bounded Variation}

A function $\mathrm{f}(\mathrm{t})$ is said to have bounded variation on $[0, T]$ if:

$$
V_{1}(f, 0, T)=\sup _{P_{n}} \sum_{j=1}^{n}\left|f\left(t_{j}\right)-f\left(t_{j-1}\right)\right|<\infty
$$

with the sup over all partitions $P_{n}, n \geq 2$.

\subsubsection{Bounded Variation and Bounded Functions}

Theorem 4.3 A function of Bounded Variation is Bounded.

So, an unbounded function can not have bounded variation, but not all function paths which do not have bounded variation are unbounded functions or there are some bounded functions which still may have unbounded variations. As we will see that Brownian motion paths do not have bounded variation but they can be bounded.

\footnotetext{
${ }^{2}$ Details on modes of convergence are in appendix
} 


\subsubsection{Bounded Variation and Monotonically Increasing Functions}

If we recall the section where we defined Stieltjes integral with either the integrator or the integrand being a function of bounded variation, we found the following relationshp between bounded variation and monotonically increasing functions:

Theorem 2.12. A function of bounded variation is equivalent to a difference of two monotonically increasing functions.

Theorem 2.13: Monotonically increasing implies bounded variation.

\subsubsection{Bounded Variation and Differentiable Functions}

Lemma 4.2 Given a continuous function $f(t)$ with the derivative $f^{\prime}(t)$ defined everywhere on $[0, T]$ and $\int_{0}^{T}\left|f^{\prime}(t)\right| d t<\infty$, then

$V_{1}(f, 0, T)=\lim _{\|\Pi\| \rightarrow 0} \sum_{j=0}^{n-1}\left|f\left(t_{j+1}\right)-f\left(t_{j}\right)\right|=\lim _{\|\Pi\| \rightarrow 0} \sum_{j=0}^{n-1}\left|f^{\prime}(t)\right|\left(t_{j+1}-t_{j}\right)=\int_{0}^{T}\left|f^{\prime}(t)\right| d t<\infty$.

Therefore, we conclude the Theorem 4.4 as follows:

Theorem 4.4 Bounded Differentiability implies Bounded Variation.

So, a roughly smooth differentiable functional path should have a bounded variation, whose path will not look like a Brownian motion's path shown in the image, Figure 1, which is nowhere differentiable almost surely, very volatile. Therefore, a functional path of a Brownian Motion which is nowhere-differentiable may not have bounded variation, and later we show that this is indeed the case.

Firstly, a relationship between monotone increasing functions and differentiable functions is provided in Junge (2004), and the same for functions of bounded variation then follows naturally.

Theorem 4.5 Let function $f(t)$ be a monotone increasing function, then $f(t)$ is differentiable almost everywhere. 


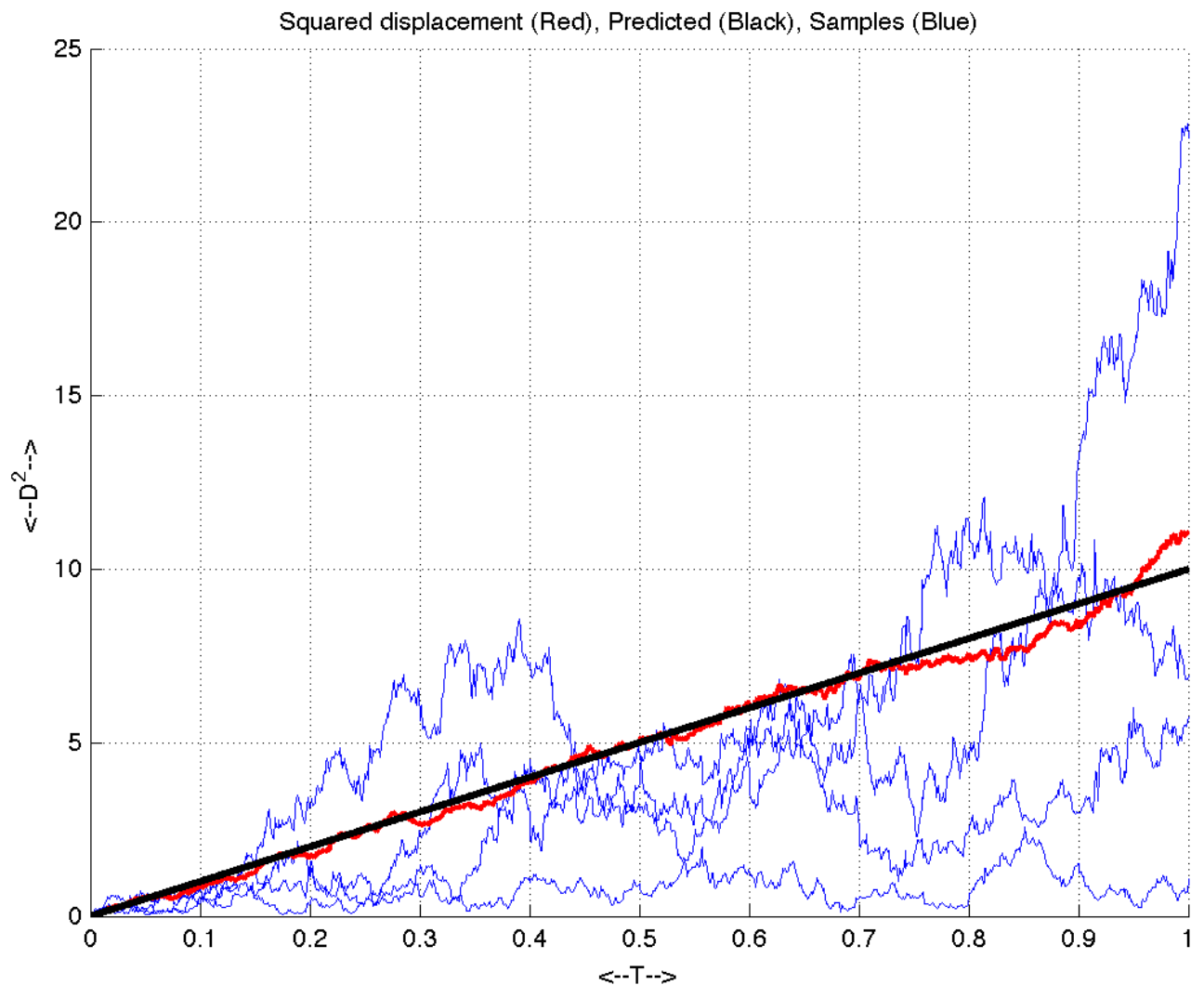

Figure 1: Brownian Motions with almost surely nowhere-differentiable paths 
Corollary 4.1 Bounded Variation implies Differentiability almost everywhere.

\section{Bounded Variation, Differentiability and Lipschitz Condition}

Generally, the following well-known theorems hold, as introduced in Labute's (2003):

\section{Sufficient conditions of Lipschitz Continuous:}

1. An everywhere differentiable function $\alpha(t)$ with bounded derivatives is Lipschitz continuous satisfying: $|\alpha(y)-\alpha(x)|<M|y-x|$ for $\forall \mathrm{x}, \mathrm{y} \in[a, b]$.

2. Bounded variation implies Lipschitz continuous.

\section{Necessary Conditions of Lipschitz Continuous:}

1. Lipschitz continuous implies bounded variation

Theorem 4.6 If a function $\alpha(t)$ satisfies Lipschitz condition such as $|\alpha(y)-\alpha(x)|<$ $M|y-x|$ for $\forall x, y \in[a, b]$, then $\alpha(t)$ has bounded variation.

2. Lipschitz continuous implies differentiable almost everywhere.

3. Lipschitz continuous needs NOT be differentiable everywhere.

\subsubsection{Brownian Motion has Unbounded Variation Almost Surely}

Lemma 4.3 Consider a continuous function $C(\omega, t)$ with bounded variation defined on $t \in$ $[0, T]$, then the sum of squared increments $Q_{n}$ over a partition $v_{n}=\left\{0=t_{0}, \ldots, t_{n}=T\right\}$ converges to 0 .

PROOF. If $C(\omega, t)$ is a continuous function in $t$ with bounded variation, in other words, 
$\sup _{v_{n}} \sum_{i=1}^{n}\left|C\left(\omega, t_{i}\right)-C\left(\omega, t_{i-1}\right)\right|<\infty$, then $\max _{i}\left|C\left(\omega, t_{i}\right)-C\left(\omega, t_{i-1}\right)\right|$ exists. Hence,

$$
\begin{aligned}
Q_{n}(T) & =\sum_{i=1}^{n}\left|C\left(\omega, t_{i}\right)-C\left(\omega, t_{i-1}\right)\right|^{2} \\
& \leq \max _{i}\left|C\left(\omega, t_{i}\right)-C\left(\omega, t_{i-1}\right)\right| \sum_{i=1}^{n}\left|C\left(\omega, t_{i}\right)-C\left(\omega, t_{i-1}\right)\right| \\
& \leq \max _{i}\left|C\left(\omega, t_{i}\right)-C\left(\omega, t_{i-1}\right)\right| \sup _{v_{n}} \sum_{i=1}^{n}\left|C\left(\omega, t_{i}\right)-C\left(\omega, t_{i-1}\right)\right|
\end{aligned}
$$

Since $C(\omega, t)$ is a continuous function in $t$, it is also uniformly continuous on $[0, T]$, hence $\max _{i}\left|C\left(\omega, t_{i}\right)-C\left(\omega, t_{i-1}\right)\right| \rightarrow 0$ as $\operatorname{mesh}\left(v_{n}\right) \rightarrow 0$. Therefore, $Q_{n}(T) \rightarrow 0$ for those $\omega$ such that $C(\omega, t)$ has bounded variation.

Theorem 4.7 Brownian motion has unbounded variation almost surely.

Mathematically, as described in Mikosch's (1998), it means,

$$
\sup _{P_{n}} \sum_{j=1}^{n}\left|B\left(\omega, t_{j}\right)-B\left(\omega, t_{j-1}\right)\right|<\infty
$$

only happens on a null-set, namely,

$$
P\left\{\omega: \sup _{P_{n}} \sum_{j=1}^{n}\left|B\left(\omega, t_{j}\right)-B\left(\omega, t_{j-1}\right)\right|<\infty\right\}=0
$$

with sup over all partitions $P_{n}$.

ProOf. Consider a Brownian Motion $B(\omega, t)$ when $t \in[0, T]$ and the set $\{\omega\} \in D$ on which Brownian Motion $B(\omega, t)$ is of bounded variation. Then, $Q_{n} \rightarrow 0$ on set $\{\omega\} \in D$ as shown in Lemma 4.3 that continuous function with bounded variation has quadratic variation converging to 0. However, for Brownian motion, $Q_{n} \rightarrow{ }^{P} T$, which implies that for appropriate subsequences $n_{k}, Q_{n_{k}} \rightarrow^{a . s .} T$ (by Theorem 4.2), in turn it means $Q_{n} \rightarrow 0$ only on a null-set.

Many details of the above lemmas can be seen in Chung (2001). 


\subsection{Brownian Motion in Contrast to a Smooth Function in Stan- dard Calculus}

Generally, the smooth function we encountered before in standard calculus has differentiable functions in it, and as we know:

- bounded differentiability implies bounded variation

- bounded variation implies differentiability almost everywhere

- continuous function of bounded variation implies 0 quadratic variation

therefore, the smooth functions are of bounded variation and 0 quadratic variation, which Brownian motion is distinguished from.

\subsection{Itô Integral Defined for Random Step Process}

A filtration is given by an increasing family of $\sigma$-algebras of events, the Brownian filtration $\left\{\mathcal{F}_{t}\right\}_{t \geq 0}$ is the smallest filtration such that $\mathcal{F}_{t}$ contains all events of the form $\left\{B_{s} \leq x\right\}$ with $0 \leq s \leq t$ and $x \in \mathbb{R}$ (see Appendix $E$ for details). In general, we will want to construct the Itô integral of $\left\{\mathcal{F}_{t}\right\}$-adapted functions in a Hilbert space $\mathcal{H}$ defined by:

$$
\mathcal{H}_{0}^{2}[0, T]:=\left\{f:[0, T] \times \Omega \rightarrow \mathcal{R}:\|f\|_{\mathcal{H}^{2}}=E\left[\int_{0}^{T} f^{2}(t) d t\right]<\infty\right\} .
$$

We first consider a dense subset of this space which includes all simple functions.

\subsubsection{Definition}

Given a partition $\Pi=\left\{t_{0}, t_{1}, \ldots, t_{n}\right\}$ on the time interval $[0, T], f_{n}(t)$ is a random step process when $f_{n}(t)$ is the constant $f_{n}\left(t_{j}\right)$ with respect to $t$ on the closed-open half interval $\left[t_{j}, t_{j+1}\right)$, then the Itô integral $I\left(f_{n}(t)\right)$ is defined as in Shreve (2004): 


\section{Definition 4.2}

$$
I\left(f_{n}(t)\right)=\sum_{j=0}^{k-1} f_{n}\left(t_{j}\right)\left[B\left(t_{j+1}\right)-B\left(t_{j}\right)\right]+f_{n}\left(t_{k}\right)\left[B(t)-B\left(t_{k}\right)\right]
$$

if $t \in\left[t_{k}, t_{k+1}\right)$.

We denote this Itô integral in definition 4.2 for step process $f_{n}(t)$ as $I\left[f_{n}(t)\right]=$ $\int_{0}^{t} f_{n}(u) d B(u)$. In particular, when $t=t_{n}=T, I\left[f_{n}(T)\right]=\int_{0}^{T} f_{n}(u) d B(u)$.

\subsubsection{Other Properties of Itô Integral Defined on Simple Processes}

As introduced in Coelen's (2002) and Øksendal's (1998), the Itô integral defined on simple processes as a random variable has the following properties:

1. Linearity: $I\left[a f_{n}(t)+b g_{n}(t)\right]=a I\left[f_{n}(t)\right]+b I\left[g_{n}(t)\right]$, in other words,

$$
\int_{0}^{T}\left[a f_{n}(t)+b g_{n}(t)\right] d B(t)=a \int_{0}^{T} f_{n}(t) d B(t)+b \int_{0}^{T} g_{n}(t) d B(t) .
$$

2. Zero mean: $E I\left[f_{n}(t)\right]=0$ or $E \int_{0}^{T} f_{n}(t) d B(t)=0$.

\subsubsection{Itô Isometry}

Itô isometry describes the relationship between the norm of $I\left[f_{n}(t)\right]$ and the norm of $f_{n}(t)$ as the following:

\section{Theorem 4.8}

$$
E\left[I^{2}\left(f_{n}(t)\right)\right]=E\left[\int_{0}^{t} f_{n}^{2}(u) d u\right]=\|f\|_{\mathcal{H}^{2}}
$$




\subsection{Itô Integral Defined for General $\mathcal{H}_{0}^{2}$ Integrands}

As we recall in the previous section, in general, the Itô integral can not be defined as a path-wise limit of the Riemann-Stieltjes sums. Take the integral form $\int_{0}^{t} B(u) d B(u)$ as an example.

Hence as introduced in Thomas Mikosch's [19], we define the Itô integral as the Mean Square Limit of the suitable Stieltjes Sums instead of the Euclidean convergence in Stieltjes Integral.

For the integrand that is a general random process $f(t) \in \mathcal{H}_{0}^{2}$, we firstly impose some assumptions on it, namely, they are as in Mikosch's (1998):

- $f(t)$ is a function of the Brownian motion $B(s), s \leq t$, i.e. adapted to the Brownian filtration, $\left\{\mathcal{F}_{t}\right\}_{t \geq 0}$.

- $\int_{0}^{t} E f^{2}(s) d t<\infty$.

As a special case, non-random continuous functions $f(s)$ defined on $[0, t]$ satisfy the above assumptions.

\subsubsection{Construction of the general Itô Integral}

For a $\mathcal{H}_{0}^{2}$ function that satisfies the above assumptions, we construct the Itô integral in the following steps:

1. Construct a Stieltjes sum for a step function $f_{n}(t)$ on a given partition $\{0=$ $\left.t_{0}, t_{1}, \ldots, t_{n}=t\right\}$ of $[0, t]: \sum_{i=0}^{n-1} f_{n}\left(t_{i}\right)\left[B\left(t_{i+1}\right)-B\left(t_{i}\right)\right]$, where $f_{n}(t)=f\left(t_{i}\right)$ when $t \in\left[t_{i}, t_{i+1}\right)$, we denote this Stieltjes sum as $I\left[f_{n}(t)\right]$.

We claim:

(a) the step function $f_{n}(t)$ converges to $f(t)$ in $\mathcal{H}_{0}^{2}$-Norm

(b) Itô integral on the step function $\left\{I_{f_{n}}(t)\right\}$ forms a Cauchy sequence in $\mathcal{H}_{0}^{2}$ and converges in $\mathcal{H}_{0}^{2}$-norm to a limit. 
2. Take the Mean Square Limit of $I\left[f_{n}(t)\right]$

The Itô integral is defined to be this unique Mean Square $\mathcal{H}_{0}^{2}$ limit and denote it as $I_{f}(t)=\int_{0}^{t} f(s) d B(s)$. Note that this limit is unique, that is, it is independent of the choice of sequence of integrands $f_{n}(t)$.

We further elaborate the following claims:

Theorem 4.9 As long as a $\mathcal{H}_{0}^{2}$ function $f(t)$ satisfies the assumptions for constructing the Itô integral, then one can find a sequence of step functions $f_{n}(t) \in \mathcal{H}_{0}^{2}$ converging in Mean Square to $f(t)$ as:

$$
E\left[\int_{0}^{t}\left(f_{n}(s)-f(s)\right)^{2} d s\right] \rightarrow 0
$$

We approximate an arbitrary function $f$ in $\mathcal{H}_{0}^{2}$ by a sequence of simple functions in $\mathcal{H}_{0}^{2}$ in $\mathcal{H}^{2}$-Norm using the following steps:

1. For any function $f \in \mathcal{H}_{0}^{2}$, there exists a bounded sequence $\left\{h_{n}\right\}$ in $\mathcal{H}_{0}^{2}$ converging to $f$ in $\mathcal{H}_{0}^{2}$-Norm.

2. For any bounded $\mathcal{H}_{0}^{2}$ function $h$, there exists a sequence of bounded continuous functions $g_{n}$ in $\mathcal{H}_{0}^{2}$ converging to $h$ in $\mathcal{H}_{0}^{2}$-Norm.

3. For any bounded continuous $\mathcal{H}_{0}^{2}$ function $g$, there exists a sequence of simple functions $\phi_{n}$ in $\mathcal{H}_{0}^{2}$ converging to $g$ in $\mathcal{H}_{0}^{2}$-Norm.

The above 3 steps of approximation in $\mathcal{H}_{0}^{2}$-Norm of an arbitrary function in $\mathcal{H}_{0}^{2}$ by simple functions in $\mathcal{H}^{2}$ can be found in page 27, Øksendal (2003).

So, after we have found the converging sequence of step functions $f_{n}(t)$ to $f(t)$ in $\mathcal{H}_{0}^{2}$-Norm, we will firstly give the proof that the limit $\mathcal{H}_{0}^{2}$-convergence of $I\left[f_{n}(t)\right]$ exists and its relationship with $I[f(t)]$ in the following:

Theorem 4.10 Given an arbitrary $\mathcal{H}_{0}^{2}$ function $f(t)$ that satisfies the assumptions for constructing an Itô integral and a sequence of step functions $\left\{f_{n}(t)\right\}$ that converges in mean 
square $\mathcal{H}_{0}^{2}$ to $f(t)$, the Ito integral defined on simple process $f_{n}(t), I_{f_{n}}(t)$ converges in mean square $\mathcal{H}_{0}^{2}$ to a unique limit in the following sense as introduced in Thomas Mikosch's (1998):

$$
E\left\{\sup _{0 \leq s \leq t}\left[I_{f_{n}}(s)-I_{f}(s)\right]^{2}\right\} \rightarrow 0
$$

PROOF. Since the sequence of Itô integrals on simple process $\left\{I_{f_{n}}(t)\right\}$ are $\mathcal{H}_{0}^{2}$ objects, we know that they converge if they form a Cauchy sequence in $\mathcal{H}_{0}^{2}$, and we say that this is in fact the case. Hence we provide the following lemma 4.4 explaining why they form a Cauchy sequence.

Lemma 4.4 Itô integrals on the simple processes $\left\{I_{f_{n}}\right\}$ form a Cauchy sequence in $\mathcal{H}_{0}^{2}$.

PROOF. The definition of Cauchy sequence says the following:

if $\forall \epsilon>0, \exists N>0$ such that $\forall m, n>N,\left\|I_{f_{n}}-I_{f_{m}}\right\| \leq \epsilon$.

And we see that this is indeed the case:

$\left\|I\left[f_{n}\right]-I\left[f_{m}\right]\right\|_{\mathcal{H}^{2}}=\left\|I\left[f_{m}-f_{n}\right]\right\|$ by the linear property of Itô integral on simple process, and

||I $\left[f_{m}-f_{n}\right]\|=\| f_{m}-f_{n} \|$ by Itô Isometry.

It does not matter if $f_{n}$ itself is a Cauchy sequence too, but $\left\|f_{m}-f_{n}\right\|=\| f_{m}-f+$ $f-f_{n}\|\leq\| f_{m}-f\|+\| f_{n}-f \|$ and this $f$ is the function that the simple process $\left\{f_{n}\right\}$ is converging to in $\mathcal{H}^{2}$, hence we can make $\left\|f_{m}-f\right\|$ and $\left\|f_{n}-f\right\|$ arbitrarily small, say each smaller than $\frac{\epsilon}{2}$ for $m, n>N$.

The idea of the above proof can be seen in Brzezniak's (1999).

Lemma 4.5 All Cauchy sequences in $\mathcal{H}_{0}^{2}$ converge to a limit in $\mathcal{H}_{0}^{2}$.

\subsubsection{Summary of the Steps to Construct the Itô Integral}

1. Define Itô integral on simple process in a legitimate subset of $\mathcal{H}_{0}^{2}$ as Stieltjes Sums. 
2. Prove any arbitrary $f(t)$ in $\mathcal{H}^{2}$ can be approximated by simple processes $f_{n}(t)$ in $\mathcal{H}^{2}$ through 3 steps:

arbitrary function $\leftarrow$ bounded function $\leftarrow$ continuous bounded function $\leftarrow$ simple function. The last step can be seen in theorems of Appendix B.2.

- Simple process $f_{n}(t)$ is constructed by discretizing the time interval $[0, t]$ into smaller time intervals as $\left[t_{j}, t_{j+1}\right), j=1, \ldots, n-1$, and on $\left[t_{j}, t_{j+1}\right)$, $f_{n}(t)$ is a constant and equals to the value of the left end-point $f\left(t_{j}\right)$.

- $f_{n}(t)$ as constructed above converges to $f(t)$ in $\mathcal{H}^{2}$ in the sense of $\lim _{n \rightarrow \infty} E \int_{0}^{T} \mid f_{n}(t)-$ $\left.f(t)\right|^{2} d t=0$.

3. $I\left[f_{n}(t)\right]$ converges to a limit in $\mathcal{H}^{2}$ because:

(a) $I\left[f_{n}(t)\right]$ forms a Cauchy sequence in $\mathcal{H}_{0}^{2}$

(b) And all Cauchy sequence in $\mathcal{H}_{0}^{2}$ converges to a limit.

And we define this limit as the Itô integral of $f(t)$, in other words,

$$
I[f(t)]=\int_{0}^{t} f(u) d B(u)=\lim _{n \rightarrow \infty} \int_{0}^{t} f_{n}(u) d B(u)
$$

in $\mathcal{H}_{0}^{2}$, where $\left\|f_{n}-f\right\|_{\mathcal{H}_{0}^{2}} \rightarrow 0$

\subsubsection{Itô Isometry}

Theorem 4.11 Assume that $f \in \mathcal{H}^{2}$. Then

$$
E I^{2}[f(t)]=E \int_{0}^{t} f^{2}(u) d u
$$

\subsection{Differences between Itô Integral and Riemann Integral}

1. Type of Convergence 
2. Choice of point in time intervals

Examples:

(a)

$$
\lim _{n \rightarrow+\infty} \sum_{j=0}^{n-1} W\left(t_{j}^{n}\right)\left[W\left(t_{j+1}^{n}\right)-W\left(t_{j}^{n}\right)\right]=\int_{0}^{T} W(t) d W(t)
$$

is an Ito Integral

(b)

$$
\lim _{n \rightarrow+\infty} \sum_{j=0}^{n-1} W\left(t_{j+1}^{n}\right)\left[W\left(t_{j+1}^{n}\right)-W\left(t_{j}^{n}\right)\right] \neq \int_{0}^{T} W(t) d W(t)
$$

is not an Ito Integral. 


\section{Itô-Doeblin Formula}

In Itô calculus, the Itô-Doeblin formula gives us the tool equivalent to the chain rule in standard calculus to derive a differential of a time-dependent composite function of a stochastic process. If the stochastic process is expressed using a stochastic differential equation, after applying Itô-Doeblin formula, we can get the differential of the composite function of the process, hence the expression in stochastic differential equation of it. To prove Itô-Doeblin formula, we use Taylor expansion on the increments of the composite function, which involves quadratic variation of the processes, hence in this section we start from introducing the quadratic variation of Brownian motion, Itô processes and jump-diffusion processes.

\subsection{Quadratic Variation}

\subsubsection{Quadratic Variation of a Brownian Motion}

As we have seen earlier, the Quadratic Variation of a Brownian Motion is

$$
[B, B](t)=\lim _{n \rightarrow \infty} \sum_{k=0}^{n-1}\left[B\left(t_{k+1}\right)-B\left(t_{k}\right)\right]^{2}=T
$$

in $\mathcal{H}^{2}$.

\subsubsection{Quadratic Variation of an Itô Integral}

1. When Itô integral is defined on a step process $f_{n}(t)$ being a constant $f_{n}\left(t_{j}\right)$ on the subinterval $\left[t_{j}, t_{j+1}\right)$ for $j=0, \ldots, n-1$.

The Itô integral $I\left[f_{n}(t)\right]$ has the quadratic variation accumulated up to time $t$ as introduced in Shreve (2004):

Theorem 5.1

$$
[I, I](t)=\int_{0}^{t} f_{n}^{2}(u) d u
$$


And as a side comparison, we can recall from Itô isometry, the second moment of Itô integral , $E\left[I^{2}\right]=E\left[\int_{0}^{t} f_{n}^{2}(u) d u\right]$.

Proof. We first look at the time interval $\left[t_{j}, t_{j+1}\right)$, the function $f_{n}(t)$ is constant on this time interval. We discretize further this time interval into $\left[t_{j}=s_{0}, s_{1}, \ldots, s_{m}=t_{j+1}\right)$, and the quadratic variation of $I\left(f_{n}(t)\right)$ accumulated on this time interval is:

$$
[I, I]=\sum_{k=0}^{m-1}\left[I\left(f_{n}\left(s_{k+1}\right)\right)-I\left(f_{n}\left(s_{k}\right)\right)\right]^{2}
$$

To simplify the notation, we denote $I\left(f_{n}\left(s_{k+1}\right)\right)$ as $I\left(s_{k+1}\right)$ and so on.

$\left[I\left(s_{k+1}\right)-I\left(s_{k}\right)\right]^{2}=f_{n}\left(t_{j}\right)\left[B\left(s_{k+1}\right)-B\left(s_{k}\right)\right]^{2}=f_{n}^{2}\left(t_{j}\right)\left[B\left(s_{k+1}\right)-B\left(s_{k}\right)\right]^{2}$

Then, $\sum_{k=0}^{m-1}\left[I\left(f_{n}\left(s_{k+1}\right)\right)-I\left(f_{n}\left(s_{k}\right)\right)\right]^{2}=f_{n}^{2}\left(t_{j}\right) \sum_{k=0}^{m-1}\left[B\left(s_{k+1}\right)-B\left(s_{k}\right)\right]^{2}$, and as $\mathrm{m}$ goes to $\infty, \sum_{k=0}^{m-1}\left[B\left(s_{k+1}\right)-B\left(s_{k}\right)\right]^{2}$ is the quadratic variation of Brownian motion, which equals to $t_{j+1}-t_{j}$, hence the quadratic variation accumulated by $I\left(f_{n}(t)\right)$ on time interval $\left[t_{j}, t_{j+1}\right)$ is $f_{n}^{2}\left(t_{j}\right)\left(t_{j+1}-t_{j}\right)$.

Then, we look at the entire time interval $\left[t_{0}=0, t=t_{n}\right)$, we sum the quadratic variation of each of the interval $\left[t_{j}, t_{j+1}\right)$, and take the limit as $n$ goes to $\infty$, this is just the Lebesgue integral for the step function $f_{n}^{2}(t)$ on a particular path, namely, $\int_{0}^{t} f_{n}^{2}(u) d u$.

Note: the quadratic variation of Itô integral $I\left[f_{n}(t)\right], \int_{0}^{t} f_{n}^{2}(u) d u$ depends on the path of $f_{n}(u)$, and therefore random.

2. When Itô integral is defined on the general adapted $\mathcal{H}^{2}$ process $f(t)$ In other words, the Itô integral $I(f(t))=\int_{0}^{t} f(t) d B(t)$, then the Quadratic Variation is:

$$
[I, I](t)=\int_{0}^{t} f^{2}(u) d u
$$




\subsubsection{Quadratic Variation of a continuous Itô Process}

Definition 5.1 A continuous Itô process $X(t)$, is defined to be a sum of a Riemann-Stieltjes integral and a Itô integral plus an initial condition, namely,

$$
X(t)=X(0)+\int_{0}^{t} \Delta(u) d B(u)+\int_{0}^{t} \Theta(u) d u
$$

The quadratic variation of such a process is given by (see Shreve (2004):

\section{Theorem 5.2}

$$
[X, X](t)=\int_{0}^{t} \Delta^{2}(u) d u
$$

The Itô-Doeblin lemma performs the "chain rule" in Itô calculus, whereas noting that Brownian motion $B(t)$ is not differentiable the usual chain rule $\frac{d}{d t} f(B(t))=$ $f^{\prime}(B(t)) B^{\prime}(t) d t$ is not valid.

\subsubsection{Quadratic Variation of a Jump Process}

Definition 5.2 Given a partition $\Pi=\left\{t_{0}, t_{1}, \ldots, t_{n}\right\}$ of $[0, T]$, the Quadratic Variation of the jump process $X(t),[X, X](t)$ is as introduced in Shreve's (2004) book in the following:

$$
[X, X](t)=\lim _{n \rightarrow \infty} \sum_{j=0}^{n-1}\left[X\left(t_{j+1}\right)-X\left(t_{j}\right)\right]^{2}
$$

where the jump process $X(t)=X(0)+X^{c}(t)+J(t)$, in which $X^{c}(t)=R(t)+I(t)=$ $\int_{0}^{t} \Theta(u) d u+\int_{0}^{t} \Gamma(u) d B(u)$ and $J(t)$ is a pure jump process.

Definition 5.3 Given two jump processes $X_{1}(t)$ and $X_{2}(t)$, the Cross Variation $\left[X_{1}, X_{2}\right](t)$ is defined as follows:

$$
\left[X_{1}, X_{2}\right](t)=\lim _{n \rightarrow \infty} \sum_{j=0}^{n-1}\left[X_{1}\left(t_{j}+1\right)-X_{1}\left(t_{j}\right)\right]\left[X_{2}\left(t_{j}+1\right)-X_{2}\left(t_{j}\right)\right]
$$


The Cross Variation of 2 jump processes is evaluated in the following result as mentioned in Shreve (2004):

\section{Theorem 5.3}

$$
\left[X_{1}, X_{2}\right](T)=\left[X_{1}^{c}, X_{2}^{c}\right](T)+\left[J_{1}, J_{2}\right](T)=\int_{0}^{T} \Gamma_{1}(s) \Gamma_{2}(s) d s+\sum_{0<s \leq T} \Delta J_{1}(s) \Delta J_{2}(s)
$$

\section{Corollary 5.1}

$$
\left[X_{1}, X_{1}\right](T)=\left[X_{1}^{c}, X_{1}^{c}\right](T)+\left[J_{1}, J_{1}\right](T)=\int_{0}^{T}\left[\Gamma_{1}(s)\right]^{2} d s+\sum_{0<s \leq T}\left[\Delta J_{1}(s)\right]^{2} .
$$

Up to here, we introduced of quadratic variations of all Brownian motion, Itô integral, continuous Itô process, Jump-Diffusion process are completed, and we are ready to investigate the Itô-Doeblin formula for all of the mentioned processes.

\subsection{Itô Formula for Brownian Motion}

Theorem $5.4 f(t, x)$ is a function such that the partial derivatives $f_{t}^{\prime}(t, x), f_{x}^{\prime}(t, x)$ and $f_{x x}^{\prime \prime}(t, x)$ are defined and continuous; $B(t)$ is a Brownian motion, then for every $T>0$ as shown in Shreve's (2004):

$$
\begin{gathered}
f(T, B(T))=f(0, B(0)) \\
+\int_{0}^{T} f_{t}^{\prime}(t, B(t)) d t+\int_{0}^{T} f_{x}^{\prime}(t, B(t)) d B(t)+\int_{0}^{T} \frac{1}{2} f_{x x}^{\prime \prime}(t, B(t)) d t
\end{gathered}
$$

or in a short form, denoted as

$$
d f(t, B(t))=f_{t}^{\prime}(t, B(t)) d t+f_{x}^{\prime}(t, B(t)) d B(t)+\frac{1}{2} f_{x x}^{\prime \prime}(t, B(t)) d t
$$

PROOF. Assume $f(t, x)$ is a general function with continuous partial derivatives. In order to obtain $f(T, B(T))$, we discretize the time interval $[0, T]$ into partitions $[0=$ 
$\left.t_{0}, t_{1}, \ldots, t_{n}=T\right]$, then $f(T, B(T))=f(0, B(0))+\sum_{0}^{n-1}\left[f\left(t_{k+1}, B\left(t_{k+1}\right)\right)-f\left(t_{k}, B\left(t_{k}\right)\right)\right]$.

If we use Taylor's expansion on the term $\left[f\left(t_{k+1}, B\left(t_{k+1}\right)\right)-f\left(t_{k}, B\left(t_{k}\right)\right)\right]$, then it is

$$
\begin{aligned}
& f_{t}^{\prime}\left(t_{k}, B\left(t_{k}\right)\right)\left(t_{k+1}-t_{k}\right)+f_{x}^{\prime}\left(t_{k}, B\left(t_{k}\right)\right)\left[B\left(t_{k+1}\right)-B\left(t_{k}\right)\right]+\frac{1}{2} f_{t t}^{\prime \prime}\left(t_{k}, B\left(t_{k}\right)\right)\left(t_{k+1}-t_{k}\right)^{2} \\
& +\frac{1}{2} f_{x x}^{\prime \prime}\left(t_{k}, B\left(t_{k}\right)\right)\left[B\left(t_{k+1}\right)-B\left(t_{k}\right)\right]^{2}+f_{t x}^{\prime \prime}\left(t_{k}, B\left(t_{k}\right)\right)\left(t_{k+1}-t_{k}\right)\left[B\left(t_{k+1}\right)-B\left(t_{k}\right)\right] .
\end{aligned}
$$

And then we take a sum for $k$ from 0 to $n-1$ on equation 34 , and further take the limit for $n$ goes to $\infty$,

$$
\begin{gathered}
\lim _{n \rightarrow \infty} \sum_{k=0}^{n-1} f_{t}^{\prime}\left(t_{k}, B\left(t_{k}\right)\right)\left(t_{k+1}-t_{k}\right)=\int_{0}^{T} f_{t}^{\prime}(t, B(t)) d t \\
\lim _{n \rightarrow \infty} \sum_{k=0}^{n-1} f_{x}^{\prime}\left(t_{k}, B\left(t_{k}\right)\right)\left[B\left(t_{k+1}\right)-B\left(t_{k}\right)\right]=\int_{0}^{T} f_{x}^{\prime}(t, B(t)) d B(t) .
\end{gathered}
$$

For the term $\lim _{n \rightarrow \infty} \sum_{k=0}^{n-1} \frac{1}{2} f_{t t}^{\prime \prime}\left(t_{k}, B\left(t_{k}\right)\right)\left(t_{k+1}-t_{k}\right)^{2}$, we have

$\left|\frac{1}{2} f_{t t}^{\prime \prime}\left(t_{k}, B\left(t_{k}\right)\right)\left(t_{k+1}-t_{k}\right)^{2}\right| \leq \frac{1}{2} f_{t t}^{\prime \prime}\left(t_{k}, B\left(t_{k}\right)\right)\left|t_{k+1}-t_{k}\right| \max _{0 \leq k \leq n-1}\left|t_{k+1}-t_{k}\right|$. Hence,

$\lim _{n \rightarrow \infty} \sum_{k=0}^{n-1}\left|\frac{1}{2} f_{t t}^{\prime \prime}\left(t_{k}, B\left(t_{k}\right)\right)\left(t_{k+1}-t_{k}\right)^{2}\right|$

$\leq \lim _{n \rightarrow \infty} \max _{0 \leq k \leq n-1}\left|t_{k+1}-t_{k}\right| \sum_{k=0}^{n-1} \frac{1}{2} f_{t t}^{\prime \prime}\left(t_{k}, B\left(t_{k}\right)\right)\left|t_{k+1}-t_{k}\right|$

$=\lim _{n \rightarrow \infty} \max _{0 \leq k \leq n-1}\left|t_{k+1}-t_{k}\right| \lim _{n \rightarrow \infty} \sum_{k=0}^{n-1} \frac{1}{2} f_{t t}^{\prime \prime}\left(t_{k}, B\left(t_{k}\right)\right)\left|t_{k+1}-t_{k}\right|$.

We also know that,

$\lim _{n \rightarrow \infty} \max _{0 \leq k \leq n-1}\left|t_{k+1}-t_{k}\right|=0$ and $\lim _{n \rightarrow \infty} \sum_{k=0}^{n-1} \frac{1}{2} f_{t t}^{\prime \prime}\left(t_{k}, B\left(t_{k}\right)\right)\left|t_{k+1}-t_{k}\right|=\frac{1}{2} \int_{0}^{T} f_{t t}^{\prime \prime}(t, B(t)) d t$ therefore,

$$
\lim _{n \rightarrow \infty} \sum_{k=0}^{n-1} \frac{1}{2} f_{t t}^{\prime \prime}\left(t_{k}, B\left(t_{k}\right)\right)\left(t_{k+1}-t_{k}\right)^{2}=0
$$

And for the term $\lim _{n \rightarrow \infty} \sum_{k=0}^{n-1} \frac{1}{2} f_{x x}^{\prime \prime}\left(t_{k}, B\left(t_{k}\right)\right)\left[B\left(t_{k+1}\right)-B\left(t_{k}\right)\right]^{2}$,

$$
\lim _{n \rightarrow \infty} \sum_{k=0}^{n-1} \frac{1}{2} f_{x x}^{\prime \prime}\left(t_{k}, B\left(t_{k}\right)\right)\left[B\left(t_{k+1}\right)-B\left(t_{k}\right)\right]^{2}=\frac{1}{2} \int_{0}^{T} f^{\prime \prime}(t, B(t)) d t
$$




\subsection{Itô Formula for continuous Itô Process}

From Shreve (2004), it gives:

\section{Theorem 5.5}

$$
\begin{gathered}
f(T, X(T))=f(0, X(0)) \\
+\int_{0}^{T} f_{t}^{\prime}(t, X(t)) d t+\int_{0}^{T} f_{x}^{\prime}(t, X(t)) d X(t)+\frac{1}{2} \int_{0}^{T} f_{x x}^{\prime \prime}(t, X(t)) d[X, X](t)
\end{gathered}
$$

where $X(t)$ is the Itô process and $f(t, x)$ with continuous partial derivatives $f_{t}^{\prime}(t, x), f_{x}^{\prime}(t, x)$, $f_{x x}^{\prime \prime}(t, x)$.

\subsection{Itô Formula for Jump Process}

Theorem 5.6 For $X(t)$ being a jump process and $f(x)$ a function with continuous derivatives $f^{\prime}(x)$ and $f^{\prime \prime}(x), f(X(t))$ is obtained by the following from Shreve's (2004):

$$
\begin{aligned}
f(X(t))=f(X(0)) & +\int_{0}^{t} f^{\prime}(X(s)) d X^{c}(s)+\int_{0}^{t} \frac{1}{2} f^{\prime \prime}(X(s)) d X^{c}(s) d X^{c}(s) \\
& +\sum_{0<s \leq t}[f(X(s))-f(X(s-))]
\end{aligned}
$$

where $d X^{c}(s) d X^{c}(s)$ denotes the quadratic variation of the continuous part.

\subsection{Itô Formula for Multiple Jump Process}

Theorem 5.7 Given two jump processes $X_{1}(t)$ and $X_{2}(t)$ and $f\left(t, x_{1}, x_{2}\right)$, a function with continuous derivatives and partial derivatives up to second order, then $f\left(t, X_{1}(t), X_{2}(t)\right)$ is 
given as the following in Shreve's (2004)

$$
\begin{aligned}
f\left(t, X_{1}(t), X_{2}(t)\right) & =f\left(0, X_{1}(0), X_{2}(0)\right)+\int_{0}^{t} f_{t}^{\prime}\left(s, X_{1}(s), X_{2}(s)\right) d s \\
& +\int_{0}^{t} f_{x_{1}}^{\prime}\left(s, X_{1}(s), X_{2}(s)\right) d X_{1}^{c}(s)+\int_{0}^{t} f_{x_{2}}^{\prime}\left(s, X_{1}(s), X_{2}(s)\right) d X_{2}^{c}(s) \\
& +\frac{1}{2} \int_{0}^{t} f_{x_{1} x_{1}}^{\prime \prime}\left(s, X_{1}(s), X_{2}(s)\right) d X_{1}^{c}(s) d X_{1}^{c}(s) \\
& +\int_{0}^{t} f_{x_{1} x_{2}}^{\prime \prime}\left(s, X_{1}(s), X_{2}(s)\right) d X_{1}^{c}(s) d X_{2}^{c}(s) \\
& +\frac{1}{2} \int_{0}^{t} f_{x_{2} x_{2}}^{\prime \prime}\left(s, X_{1}(s), X_{2}(s)\right) d X_{2}^{c}(s) d X_{2}^{c}(s) \\
& +\sum_{0<s \leq t}\left[f\left(s, X_{1}(s)\right), X_{2}(s)-f\left(s-, X_{1}(s-)\right), X_{2}(s-)\right]
\end{aligned}
$$

Corollary 5.2 An equivalent statement of the "Product Rule" for Itô jump processes is given as the following in Shreve's (2004)

$$
\begin{aligned}
X_{1}(t) X_{2}(t) & =X_{1}(0) X_{2}(0)+\int_{0}^{t} X_{1}(s) d X_{2}^{c}(s)+\int_{0}^{t} X_{2}(s) d X_{1}^{c}(s)+\left[X_{1}^{c}, X_{2}^{c}\right](t) \\
& +\sum_{0<s \leq t}\left[X_{1}(s) X_{2}(s)-X_{1}(s-) X_{2}(s-)\right] \\
& =X_{1}(0) X_{2}(0)+\int_{0}^{t} X_{1}(s-) d X_{2}(s)+\int_{0}^{t} X_{2}(s-) d X_{1}(s)+\left[X_{1}, X_{2}\right](t) .
\end{aligned}
$$




\section{Stochastic Integral with Jump-Diffusion Processes as the Integrator}

\subsection{Itô Integral with Continuous Itô Process as the Integrator}

Definition 6.1 We define the integration of an $\mathcal{F}_{t}$-adapted process $\Gamma(t)$ with respect to an Itô process $X(u)$ as follows:

$$
\int_{0}^{t} \Gamma(u) d X(u)=\int_{0}^{t} \Gamma(u) \Delta(u) d B(u)+\int_{0}^{t} \Gamma(u) \Theta(u) d u
$$

where $X(t)=X(0)+\int_{0}^{t} \Delta(u) d B(u)+\int_{0}^{t} \Theta(u) d u$.

\subsection{Stochastic Integral with Pure Jump Process as the Integrator}

Definition 6.2 Define Stochastic Integral w.r.t. Compound Poisson Process as:

$$
\int_{0}^{T} \phi_{t} d Y_{t}:=\sum_{k=1}^{N_{t}} \phi_{t_{k}} Z_{k}=\sum_{k=1}^{N_{t}} \phi_{t_{k}}\left[Y_{t_{k}}-Y_{t_{k}-}\right]=\int_{0}^{T} \phi_{s}\left[Y_{s}-Y_{s-}\right] d N_{s}
$$

- $\left\{\phi_{t}\right\}_{t \geq 0}=a$ stochastic process

- $\left\{Y_{t}\right\}=$ the Compound Poisson Process $\sum_{k=1}^{N_{t}} Z_{k}$; where $Z_{k}$ are the jump sizes.

Special Case: Stochastic Integral w.r.t. Simple Poisson Process:

- $\left\{\phi_{t}\right\}_{t \geq 0}=$ a stochastic process

- $\left\{N_{t}\right\}=$ the Simple Poisson Process $\sum_{k=1}^{N_{t}}\left(Z_{k}=1\right)$

Definition 6.3 Define the Stochastic Integral w.r.t. Simple Poisson Process as:

$$
\int_{0}^{T} \phi_{t} d N_{t}:=\sum_{k=1}^{N_{t}} \phi_{t_{k}} Z_{k}=\sum_{k=1}^{N_{t}} \phi_{t_{k}}
$$




\subsection{Stochastic Integral with Jump-Diffusion Process as the Inte- grator}

A Jump-Diffusion process includes both continuous terms and jump terms.

Definition 6.4 Given the Jump-Diffusion process $X_{t}=\int_{0}^{t} v_{s} d s+\int_{0}^{t} u_{s} d B_{s}+Y_{t}$, where $Y_{t}$ is a pure Jump Process (Compound Poisson), we define the stochastic integral w.r.t. the general jump process $X(t)$ as follows:

$$
\int_{0}^{t} \phi(s) d X_{s}=\int_{0}^{t} \phi(s) v_{s} d s+\int_{0}^{t} \phi(s) u_{s} d B_{s}+\int_{0}^{t} \phi(s) d Y_{s}
$$

where $\int_{0}^{t} \phi(s) d Y_{s}=\sum_{k=1}^{N_{t}} \phi\left(T_{k}\right) Z_{k}=\sum_{0<s \leq t} \phi(s) \Delta Y_{s}$

Hence, the same as above:

- Any jump process is a stochastic integral w.r.t. a jump process

- A stochastic integral w.r.t. a jump process produces a jump process in turn.

\subsubsection{More on Itô-Doeblin Formula for Jump Stochastic Integral}

\section{Function of a Compound Poisson Process:}

$f\left(Y_{t}\right)$ is a function of the Compound Poisson $Y_{t}$, hence $f\left(Y_{t}\right)$ is a Jump Process too and it shares the same jump times as $Y_{t}$, therefore, it can be expressed as a Stochastic Integral of the follows:

$$
f\left(Y_{t}\right)=f\left(Y_{0}\right)+\sum_{k=1}^{N_{t}} f\left(Y_{T_{k}}\right)-f\left(Y_{T_{k}-}\right)=\int_{0}^{t}\left[f\left(Y_{s}\right)-f\left(Y_{s-}\right)\right] d N_{s} .
$$

\section{Function of a Simple Poisson Process:}

$f\left(N_{t}\right)$ is also a Jump Process, where $N_{t}$ is a simple Poisson process, therefore can be expressed as a stochastic integral, by summing the jump increments, 
we have:

$$
\begin{aligned}
f\left(N_{t}\right) & =f(0)+\sum_{k=1}^{N_{t}}[f(k)-f(k-1)] \\
& =\int_{0}^{t}\left[f\left(N_{s-}+1\right)-f\left(N_{s-}\right)\right] d N_{t} \\
& =\int_{0}^{t}\left[f\left(N_{s}\right)-f\left(N_{s-}\right)\right] d N_{t} .
\end{aligned}
$$

\section{Function of a General Itô Process:}

Ito Process $X_{t}$ with jump terms - Jump Diffusion Process

$$
\begin{aligned}
& X_{t}=X_{0}+\int_{0}^{t} v_{s} d s+\int_{0}^{t} u_{s} d B_{s}+\int_{0}^{t} \eta_{s} d Y_{s} \\
& \int_{0}^{t} \eta_{s} d Y_{s}=\sum_{k=1}^{N_{t}} \eta\left(T_{k}\right)\left[Y_{T_{k}}-Y_{T_{k}-}\right]=\sum_{k=1}^{N_{t}} \eta\left(T_{k}\right)\left[\left(Y_{T_{k}-}+Z_{k}\right)-Y_{T_{k}-}\right]=\sum_{k=1}^{N_{t}} \eta\left(T_{k}\right) Z_{k}
\end{aligned}
$$

Hence, the jump size of $X_{t}$ at jump time $T_{k}$ is: $X_{T_{k}}-X_{T_{k}-}=\eta\left(T_{k}\right) Z_{k}$. Therefore,

$$
\begin{aligned}
d f\left(X_{t}\right) & =f_{t}^{\prime} d t+f_{x}^{\prime} v_{t} d t+u_{t} f^{\prime}\left(X_{t}\right) d B_{t} \\
& +\frac{1}{2} f^{\prime \prime}\left(X_{t}\right)\left|u_{t}\right|^{2} d t+\left[f\left(X_{T_{k}-}+\eta_{T_{k}} Z_{k}\right)-f\left(X_{T_{k}-}\right)\right]
\end{aligned}
$$

or,

$$
\begin{aligned}
f\left(X_{t}\right) & =f\left(X_{0}\right)+\int_{0}^{t} f_{s}^{\prime} d s+\int_{0}^{t} f_{x}^{\prime} v_{s} d s+\int_{0}^{t} u_{s} f^{\prime}\left(X_{s}\right) d B_{s} \\
& +\frac{1}{2} \int_{0}^{t} f^{\prime \prime}\left(X_{s}\right)\left|u_{s}\right|^{2} d s+\sum_{k=1}^{N_{t}}\left[f\left(X_{T_{k}-}+\eta_{T_{k}} Z_{k}\right)-f\left(X_{T_{k}-}\right)\right] \\
& =f\left(X_{0}\right)+\int_{0}^{t} f_{s}^{\prime} d s+\int_{0}^{t} f_{x}^{\prime} v_{s} d s+\int_{0}^{t} u_{s} f^{\prime}\left(X_{s}\right) d B_{s} \\
& +\frac{1}{2} \int_{0}^{t} f^{\prime \prime}\left(X_{s}\right)\left|u_{s}\right|^{2} d s+\int_{0}^{t} f\left(X_{s}\right)-f\left(X_{s-}\right) d N_{s} .
\end{aligned}
$$




\section{General Theorem:}

$$
\begin{aligned}
f(X(t)) & =f(X(0))+\int_{0}^{t} f^{\prime}(X(s)) d X^{c}(s) \\
& +\frac{1}{2} \int_{0}^{t} f^{\prime \prime}(X(s)) d X^{c}(s) d X^{c}(s) \\
& +\sum_{0<s \leq t}[f(X(s))-f(X(s-))] .
\end{aligned}
$$

PROOF.

$\left.X(t)=X(0)+I(t)+R(t)+J(t) ; I(t)=\int_{0}^{(} t\right) \Gamma(s) d B(s) ; R(t)=\int_{0}^{t} \Theta(s) d s ;$

$X^{c}(t)=I(t)+R(t)$.

Assume the jump times are $0=\tau_{0}<\tau_{1}<\ldots<\tau_{n-1}<\tau_{n}=t$, where $\tau_{0}=0$ and $\tau_{n}=t$ are not jump times.

$f(X(t))=\sum_{j=0}^{n-1} f\left(X\left(\tau_{j+1}\right)\right)-f\left(X\left(\tau_{j}\right)\right)$.

When $u$ and $v$ are between $\tau_{j-1}$ and $\tau_{j}, X(u)=X^{c}(u)$, similarly, $X(v)=X^{c}(v)$, then, $\triangle f=f(X(v))-f(X(u))=\int_{u}^{v} f^{\prime}(X(s)) d X^{c}(s)+\frac{1}{2} \int_{u}^{v} f^{\prime \prime}(X(s)) d X^{c}(s) d X^{c}(s)$, and, $\lim _{u \rightarrow \tau_{j}} \triangle f=f(X(v))-f\left(X\left(\tau_{j}\right)\right)$ by right continuity; furthur, take

$$
\begin{aligned}
\lim _{v \rightarrow \tau_{j+1}} \triangle f & =f\left(X\left(\tau_{j+1}-\right)\right)-f\left(X\left(\tau_{j}\right)\right) \\
& =\int_{\tau_{j}}^{\tau_{j+1}} f^{\prime}(X(s)) d X^{c}(s)+\frac{1}{2} \int_{\tau_{j}}^{\tau_{j+1}} f^{\prime \prime}(X(s)) d X^{c}(s) d X^{c}(s)
\end{aligned}
$$

so

$$
\begin{aligned}
f\left(X\left(\tau_{j+1}\right)\right)-f\left(X\left(\tau_{j}\right)\right) & =f\left(X\left(\tau_{j+1}-\right)\right)-f\left(X\left(\tau_{j}\right)\right)+\left[f\left(X\left(\tau_{j+1}\right)\right)-f\left(X\left(\tau_{j+1}-\right)\right)\right] \\
& =\int_{\tau_{j}}^{\tau_{j+1}} f^{\prime}(X(s)) d X^{c}(s) \\
& +\frac{1}{2} \int_{\tau_{j}}^{\tau_{j+1}} f^{\prime \prime}(X(s)) d X^{c}(s) d X^{c}(s) \\
& +\left[f\left(X\left(\tau_{j+1}\right)\right)-f\left(X\left(\tau_{j+1}-\right)\right)\right] .
\end{aligned}
$$


Therefore,

$$
\begin{aligned}
f(X(t)) & =\sum_{j=0}^{n-1} f\left(X\left(\tau_{j+1}\right)\right)-f\left(X\left(\tau_{j}\right)\right) \\
& =\int_{0}^{\tau_{t}} f^{\prime}(X(s)) d X^{c}(s)+\frac{1}{2} \int_{0}^{\tau_{t}} f^{\prime \prime}(X(s)) d X^{c}(s) d X^{c}(s) \\
& +\sum_{j=0}^{n-1}\left[f\left(X\left(\tau_{j+1}\right)\right)-f\left(X\left(\tau_{j+1}-\right)\right)\right] .
\end{aligned}
$$

Notice that all the integrals are w.r.t. the continuous part $X^{c}(s)$

Note:

1. The reason we can use $\tau_{j+1}$ instead of $\tau_{j+1}-$ in the integral is because we are integrating w.r.t. $X^{c}(s)$ instead of the jump process $X(s)$. i.e.

$$
\lim _{v \rightarrow \tau_{j+1}} \int_{\tau_{j}}^{v} f^{\prime}(X(s)) d X(s)=\int_{\tau_{j}}^{\tau_{j+1}-} f^{\prime}(X(s)) d X(s)+\left[f\left(X\left(\tau_{j+1}\right)\right)-f\left(X\left(\tau_{j+1}-\right)\right)\right] .
$$

\subsection{S.D.E. with Jumps}

We now give two examples of stochastic differential equations driven by Poisson processes.

Examples: $\bullet d S_{t}=\eta S_{t-} d N_{t}$

$$
\begin{aligned}
& d S_{t}=S_{t}-S_{t-}=\eta S_{t-} \Rightarrow S_{t}=S_{t-}+\eta S_{t-}=(1+\eta) S_{t-} \\
& \Rightarrow S(t)=S_{0}(1+\eta)^{N_{t}}
\end{aligned}
$$

- $d S_{t}=\eta_{t} S_{t-} d N_{t}$

$$
\begin{aligned}
& d S_{t}=S_{t}-S_{t-}=\eta_{t} S_{t-} \Rightarrow S_{t}=\left(1+\eta_{t}\right) S_{t-} \\
& \Rightarrow S_{t}=S_{0} \prod_{k=1}^{N_{t}}\left(1+\eta_{T_{k}}\right), \text { where } T_{k} \text { is the jump times of the } k^{t h} \text { jump of } N .
\end{aligned}
$$




\section{Jump-Diffusion Processes in Financial Applications}

\subsection{Volatility and Quadratic Variation of Diffusion Models}

\subsubsection{Concepts of Volatility in Diffusion Models:}

Volatility is a theoretical and usually unobservable object that controls the degree of fluctuation of the rate of change process. The Volatility in diffusion models refers to either the instantaneous diffusion coefficient or the quadratic variation over a given time period - integrated volatility. The following definitions about volatility in financial settings can be seen in Visser (2009).

Definition 7.1 Given a stochastic differential equation

$$
d p(t)=\sigma(t) d B(t)
$$

where $p(t)$ is the log price process, then the variable $\sigma(t)$ is called the spot volatility or instantaneous volatility.

Further, equation (41) can be also rewritten as:

$$
p(t)=\int_{0}^{T} \sigma(t) d B(t)
$$

Hence, by Itô isometry, $E\left[p^{2}(t)\right]=E\left[\int_{0}^{T} \sigma^{2}(t) d t\right]=\int_{0}^{T} \sigma^{2}(t) d t$, and this is called the integrated volatility, we can further prove that this is also the quadratic variation of the $\log$ price process $p(t)$, namely,

$$
\sum_{i}\left[p\left(t_{i}\right)-p\left(t_{i-1}\right)\right]^{2}=\int_{0}^{T} \sigma^{2}(t) d t
$$

Usually in foreign exchange rates markets, it is assumed that the volatility process $\sigma(t)$ is independent from the Brownian motion, as a result, the log return $r_{n}=$ 
$p\left(t_{n}\right)-p\left(t_{n-1}\right)$ is normally distributed with variance $Q V_{n}: r_{n} \sim N\left(0, Q V_{n}\right)$ where $Q V_{n}$ is the quadratic variation over the $\mathrm{n}$-th period.

- Realized Volatility and Implied Volatility

The finite sample $\mathrm{n}$ steps of quadratic variation: $\sum_{i=1}^{n}\left[p\left(t_{i}\right)-p\left(t_{i-1}\right)\right]^{2}$ is called the realized volatility. An estimate of the future realized price volatility is usually the input in Black-Scholes formula to determine the theoretical value of an option such as $C=f\left(\sigma_{,}.\right)$. Hence, if we take the inverse function $g=f^{-1}$, then $\sigma_{\bar{c}}=g\left(\bar{C}_{1}.\right)$, where $\bar{C}$ is the market price of an option and $\sigma_{\bar{c}}$ is the implied volatility by the market price.

\subsection{Some Financial Instruments:}

The introduction of the following financial concepts can be seen in Coelen's (2002) and Hull's (1997).

\section{Financial Derivatives:}

A derivative is a financial instrument whose value depends on the value of other basic assets, such as stocks.

\section{Options:}

- Call option: gives the holder the right to buy an asset by a certain date at a certain price.

- European option: exercise can only be made on the maturity date.

- Put option: gives the holder the right to sell an asset by a certain date at a certain price

- Options contract has an up-front fee / price.

\section{Hedge:}

make an investment to reduce the risk of adverse price movements in an asset. 
- An example of hedging using Put Option: is described as follows:

Alan owns 100 shares of Blackberry stock on October 1, 2013. The share price is $\$ 7.92$ per share. Alan wants to avoid the risk that the share price dives in the next 2 months, so he purchased a put option that gives him the right to sell all 100 shares for a strike price of $\$ 7$. If the option price is $\$ 2$ per share, each put option that allows for the sale of 100 shares, hence costs $\$ 200$.

\section{Stock Price Modelling:}

Usually, the absolute change in the stock price does not reveal a lot of information, instead, it is the relative change of the price which is more valuable. For example, absolute changes of $\$ 1$ from $\$ 0.1$ and from $\$ 100$ matter very differently. Given the current stock price $S(t)$, we would like to consider a chance in price $d S$ during a small time interval $d t$, and the contribution to the return on the stock, $\frac{d S}{S}$ are composed by 2 parts, one part, $\mu d t$ from deterministic goverment risk-free Treasury bill with a risk free interest rate $\mu$ on the bond, where $\mu$ measures the average rate of growth of the stock - the drift; another part, $\sigma d B$, from the random changes in the stock price $S$ caused by unanticipated external effects. These random changes are best modelled by random samples drawn from a normal distribution with 0 mean and standard deviation $\sigma . \sigma$ is defined as the volatility of the stock.Therefore, putting the deterministic drift part from risk-free government Treasury bill and random change volatility part, the SDE is:

$$
\frac{d S}{S}=\mu d t+\sigma d B
$$

If there is no volatility $\sigma$, in other words, the variance of the stock is 0 , then $\frac{d S}{S}=\mu d t$ and $S(t)=S_{0} e^{\mu t}$

- The use of Brownian motion: the independent increment property of Brownian motion ensures that the price changes from now will be independent of 
the past.

\subsection{Black-Scholes-Merton Model}

\section{Backgrounds:}

Firstly, we look at a value process of a portfolio of investment, $X(t)$.

This portfolio includes 2 types of investments, namely, risk-free money market account and risky stock investment.

In the money market, there is a constant interest rate of $r$ while in the risky stock investment, the price process of the stock is modelled by a geometric brownian motion as the following SDE:

$$
d S(t)=\alpha S(t) d t+\sigma S(t) d B(t)
$$

\section{A Snap Shot at Time $t$ :}

In stock investment: the portfolio has $\Delta(t)$ shares of stocks, hence the value from the stock market is $\Delta(t) S(t)$.

In money market: the value therefore is $\beta_{t}=X(t)-\Delta(t) S(t)=\beta_{0} e^{r t}$.

Therefore, $\beta_{t}$ follows:

$$
d \beta_{t}=r \beta_{t} d t
$$

In other words, the portfolio follows the self-financing assumption from only the investment of risky stocks and non-risky bonds. We obtain a SDE for the total porfolio value process $X(t)$ as follows:

Portfolio Value Process:

$d X(t)=\Delta(t) d S(t)+r[X(t)-\Delta(t) S(t)] d t=r X(t) d t+\Delta(t)(\alpha-r) S(t) d t+\Delta(t) \sigma S(t) d B_{t}$

\section{Option Value Process:}

- European call option strike price: $(S(t)-K)^{+}$ 
- Value of the call option: $c(t, S(t))$

By Itô-Doeblin formula:

$$
\begin{gathered}
d c(t, S(t))=c_{t}(t, S(t)) d t+c_{x}(t, S(t)) d S(t)+\frac{1}{2} c_{x x}(t, S(t)) d S(t) d S(t) \\
=\left[c_{t}(t, S(t))+\alpha S(t) c_{x}(t, S(t))+\frac{1}{2} \sigma^{2} S^{2}(t) c_{x x}(t, S(t))\right] d t+\sigma S(t) c_{x}(t, S(t)) d B(t) .
\end{gathered}
$$

\section{Discounted Proces:}

Consider $e^{-r t} S(t), e^{-r t} X(t)$ and $e^{-r t} c(t, S(t))$.

By Itô-Doeblin formula, for function $f(t, x)$ :

$$
d f(t, x)=f_{t}^{\prime} d t+f_{x}^{\prime} d x+\frac{1}{2} f_{x x}^{\prime \prime} d S(t) d S(t)
$$

When $f\left(t, S_{t}\right)=e^{-r t} S(t), f_{t}^{\prime}=-r e^{-r t} S_{t}, f_{s}^{\prime}=e^{-r t}, f_{s s}^{\prime \prime}=0$, hence,

- Discounted Stock Price Process:

$$
d\left[e^{-r t} S_{t}\right]=-r e^{-r t} S_{t} d t+e^{-r t} d S_{t}=(\alpha-r) e^{-r t} S_{t} d t+\sigma S_{t} e^{-r t} d B_{t}
$$

When $f\left(t, X_{t}\right)=e^{-r t} X(t), f_{t}^{\prime}=-r e^{-r t} X_{t}, f_{x}^{\prime}=e^{-r t}, f_{x x}^{\prime \prime}=0$, hence,

- Discounted Portfolio Value Process:

$$
\begin{gathered}
d\left[e^{-r t} X(t)\right]=-r e^{-r t} X(t) d t+e^{-r t} d X(t) \\
=\Delta(t)(\alpha-r) e^{-r t} S(t) d t+\Delta(t) \sigma e^{-r t} S(t) d B(t)=\Delta(t) d\left[e^{-r t} S_{t}\right] .
\end{gathered}
$$


- Discounted Option Value Process:

$$
\begin{aligned}
d\left(e^{-r t} c(t, S(t))\right) & =e^{-r t}\left[-r c(t, S(t))+c_{t}(t, S(t))+\alpha S(t) c_{x}(t, S(t))\right. \\
& \left.+\frac{1}{2} \sigma^{2} S^{2}(t) c_{x x}(t, S(t))\right] d t+e^{-r t} \sigma S(t) c_{x}(t, S(t)) d B(t) .
\end{aligned}
$$

\section{Portfolio Value Process and Option Value Process Equation:}

We need the portfolio value $X(t)$ agrees with option value $c(t, S(t))$ at each time $t$ before the maturity date $\mathrm{T}$, which is equivalent to that the 2 discounted processes agree, namely, $e^{-r t} X(t)=e^{-r t} c(t, S(t))$, and this is further guaranteed if

$$
d\left[e^{-r t} X(t)\right]=d\left[e^{-r t} c(t, S(t))\right]
$$

When we expand the left and right hand side of euqation (47), we see that in order for the 2 sides to agree, we need as shown in Shreve's (2004):

1. $\Delta(t)=C_{x}(t, S(t))$, the number of shares held at time $t$ equals the derivative of option value process with respect to the stock price at time $t$

2. $r c(t, S(t))=c_{t}(t, S(t))+r S(t) c_{x}(t, S(t))+\frac{1}{2} \sigma^{2} S^{2}(t) c_{x x}(t, S(t))$.

Therefore, we need to find an option value process $c(t, S(t))$ that satisfies the following:

1. $r c(t, S(t))=c_{t}(t, S(t))+r S(t) c_{x}(t, S(t))+\frac{1}{2} \sigma^{2} S^{2}(t) c_{x x}(t, S(t))$

2. $X(T)=c(T, S(T))=(S-K)^{+}$

3. Delta hedging strategy: $\Delta(t)=c_{x}(t, S(t))$.

\subsection{Modelling Asset Price using Jump Processes}

In particular, we investigate an asset price that is modelled by a driving force of a Brownian motion and a compound process as introduced in Shreve's (2004): So, the 
stock's price process is modelled by the following SDE:

$$
d S(t)=(\alpha-\beta \lambda) S(t) d t+\sigma S(t) d B(t)+S(t-) d Q(t)
$$

where $Q(t)$ is a compound Poisson process and $Q(t)=\sum_{i=1}^{N(t)} Y_{i}$. We will verify that the solution for this price process is: Shreve (2004).

$$
S(t)=S(0) \exp \left\{\sigma B(t)+\left(\alpha-\beta \lambda-\frac{1}{2} \sigma^{2}\right) t\right\} \prod_{i=1}^{N(t)}\left(Y_{i}+1\right) .
$$

Proof. We set $S(0) \exp \left\{\sigma B(t)+\left(\alpha-\beta \lambda-\frac{1}{2} \sigma^{2}\right) t\right\}=X(t)$ and $\prod_{i=1}^{N(t)}\left(Y_{i}+1\right)=J(t)$, then $S(t)=X(t) J(t)$.

From Itô product rule, $X(t) J(t)=\int_{0}^{t} X(s-) d J(s)+\int_{0}^{t} J(s) d X(s)$. And since $[X(t), J(t)]=$ 0 , so this term does not appear in the product rule formula above.

From $J(t)=\prod_{i=1}^{N(t)}\left(Y_{i}+1\right)$, we can get $J(t)=J(t-)\left(Y_{i}+1\right)$.

In other words, $\Delta J(t)=J(t)-J(t-)=J(t-) Y_{i}$, and we also know $\Delta Q(t)=Y_{i}$.

Hence, $\Delta J(t)=J(t)-J(t-)=J(t-) \Delta Q(t)$ and $d J(t)=J(t-) d Q(t)$.

On the other hand, since $X(t)=S(0) \exp \left\{\sigma B(t)+\left(\alpha-\beta \lambda-\frac{1}{2} \sigma^{2}\right) t\right\}=f(t, B(t))$.

And then,

$f_{t}^{\prime}=S(0) \exp \{\sigma B(t)\} \exp \left\{\left(\alpha-\beta \lambda-\frac{1}{2} \sigma^{2}\right) t\right\}\left(\alpha-\beta \lambda-\frac{1}{2} \sigma^{2}\right)=X(t)\left(\alpha-\beta \lambda-\frac{1}{2} \sigma^{2}\right)$,

$f_{w}^{\prime}=X(t) \sigma$ and $f_{w w}^{\prime \prime}=\sigma^{2} X(t)$. Therefore,

$$
\begin{aligned}
d X(t) & =f_{t}^{\prime} d t+f_{w}^{\prime} d B(t)+\frac{1}{2} f_{w w}^{\prime \prime} d t \\
& =X(t)\left(\alpha-\beta \lambda-\frac{1}{2} \sigma^{2}\right) d t+\sigma X(t) d B(t)+\frac{1}{2} \sigma^{2} X(t) d t \\
& =X(t)(\alpha-\beta \lambda) d t+\sigma X(t) d B(t) .
\end{aligned}
$$


As a result,

$$
\begin{aligned}
X(t) J(t) & =\int_{0}^{t} X(s-) J(s-) d Q(s)+\int_{0}^{t} J(s) X(s)(\alpha-\beta \lambda) d s+\int_{0}^{t} J(s) \sigma X(s) d B(s) \\
& =\int_{0}^{t} S(u-) d Q(u)+\int_{0}^{t} S(u)(\alpha-\beta \lambda) d u+\int_{0}^{t} \sigma S(u) d B(u) .
\end{aligned}
$$

And this is the integral form of our SDE in Equation (48).

\subsubsection{Merton Jump-Diffusion Model}

$$
\frac{d S(t)}{S(t-)}=\mu d t+\sigma d B(t)+d J(t)
$$

or

$$
d S(t)=\mu S(t-) d t+\sigma S(t-) d B(t)+S(t-) d J(t)
$$

where $d J(t)=Y_{j}-1$ at jump times $\tau_{j}$.

\section{Solution:}

At jump times $\tau_{1}, \tau_{2}, \ldots, \tau_{j}$, the jump size of the price process is:

$S(t)-S(t-)=S(t-)\left(Y_{j}-1\right)$. Therefore, $S(t)=S(t-) Y_{j}$.

Hence, the jump size of the log price process is:

$$
\log S(t)=\log S(t-)+\log Y_{j}
$$

At non-jump times, $S(t)-S(t-)=0$.

Set $f\left(S_{t}, t\right)=\log S_{t}$, then $f_{t}^{\prime}=0, f_{x}^{\prime}=\frac{1}{S_{t}}, f_{x x}^{\prime \prime}=-\frac{1}{S_{t}^{2}}$, then by Itô's formula:

$$
d \log S(t)=\mu \frac{S(t-)}{S(t)} d t+\frac{\sigma S(t-)}{S(t)} d B_{t}-\frac{\sigma^{2} S(t-)^{2}}{2 S(t)^{2}} d t+\log Y_{j}
$$

In the continuous component part of equation $(49), S(t-)=S(t)$, hence the equation is reduced to

$$
d \ln S(t)=\mu d t+\sigma d B_{t}-\frac{\sigma^{2}}{2} d t+\log Y_{j}
$$


Hence,

$$
\ln S(t)=\ln S(0)+\left(\mu-\frac{\sigma^{2}}{2}\right) t+\sigma B_{t}+\sum_{j=1}^{N_{t}} \log Y_{j} .
$$

In other words,

$$
S(t)=S(0) \exp \left\{\left(\mu-\frac{\sigma^{2}}{2}\right) t+\sigma B_{t}\right\} \prod_{j=1}^{N_{t}} Y_{j} .
$$




\section{Application in Monte Carlo Simulation}

In general, Monte Carlo simulation provides a way to estimate an integral $\int_{0}^{T} f(t) d \alpha(t)$ when an analytic evaluation is not possible. Its procedure as a stochastic algorithm provides as introduced in Chorro's (2008):

- A numerical estimate of this integral,

- An estimate of the error,

- The balance between accuracy and computational cost.

To give a visual presentation of a Black-Scholes-Merton path and the Merton JumpDiffusion model path, we simulate using the discretization method and the implementation is conducted in software MATLAB. We will see that there are distinguishable outcomes especially in the QQ plots between the two models of Black-Scholes and jump-diffusion. But firstly, we will also take a look at the simulation of fractional Brownian motion paths using Euler hypergeometric integral and Gaussian quadrature.

\subsection{Fractional Brownian Motion Paths}

Fractional Brownian motion path can be simulated using exact or approximated simulation methods. In this thesis, we explore the implementation using Euler hypergeometric integrals in approximated methods as described in Cook's (2003).

\subsubsection{Euler Hypergeometric Integrals}

A hypergeometric function $F\left(a_{1}, \ldots, a_{p} ; b_{1}, \ldots, b_{q} ; z\right)$ has a power series representation

$$
\sum_{k>0} \frac{a_{1}^{\bar{k}} \ldots a_{p}^{\bar{k}} z^{k}}{b_{1}^{\bar{k}} \ldots b_{q}^{\bar{k}} k !}
$$


where $a^{\bar{k}}=a(a+1) \ldots(a+k-1)$.

And a hypergeometric function is called Gaussian when $p=2$ and $q=1$. We denote it as $F_{2,1}\left(a_{1}, a_{2} ; b_{1}, z\right)$ here. As introduced in The Gaussian hypergeometric function used in fractional Brownian motion is

$$
K_{H}(t, s)=\frac{(t-s)^{H-\frac{1}{2}}}{\Gamma(H+12)} F_{2,1}\left(H-\frac{1}{2} ; \frac{1}{2}-H ; H+\frac{1}{2} ; 1-\frac{t}{s}\right)
$$

and the $\mathrm{fBm}$

$$
B^{H}(t)=\int_{0}^{t} K_{h}(t, s) d B(s)
$$

\subsubsection{Gaussian Quadrature}

As shown in Olson's 2009, a quadrature rule to compute an integral is represented as:

$$
\int_{a}^{b} f(x) d x \approx \sum_{j=0}^{n} \omega_{j} f\left(x_{j}\right)
$$

The Gaussian quadrature chooses $\omega_{j}$ and $x_{j}$ such that the quadrature rule is exact for really high polynomials. Further, The following steps are chosen:

- Partition the interval $[a, b]$ into $a \leq x_{0}<x_{1}<\ldots<x_{n-1}<x_{n} \leq b$ where the partition nodes $\left\{x_{0}, \ldots, x_{n}\right\}$ can be chosen anywhere between a and $b$, ordered. The exact integration can be made for polynomials with degrees $2 n+1$ or less.

Paths of fractional Brownian motion with Hurst parameter 0.3 and 0.7 are exhibited in Figure 2 and 3 respectively.

\subsection{Simulation of Black-Scholes-Merton Model}

\section{Discretization of the Solution Process:}

An introduction of the following schemes can be seen in Glasserman's (2003). From 


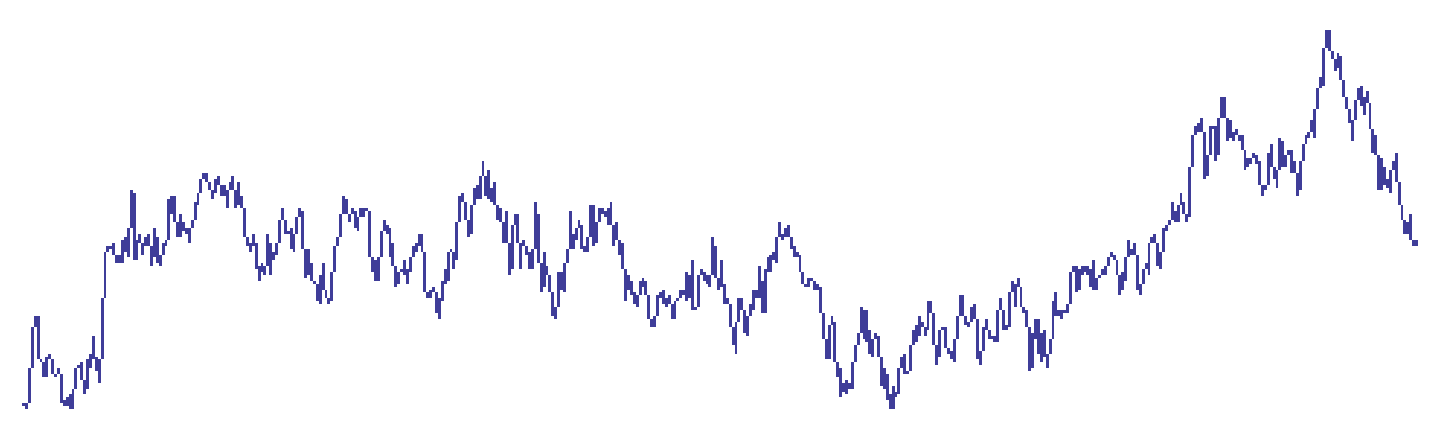

Figure 2: A Fractional Brownian Motion Path with $H=0.3$

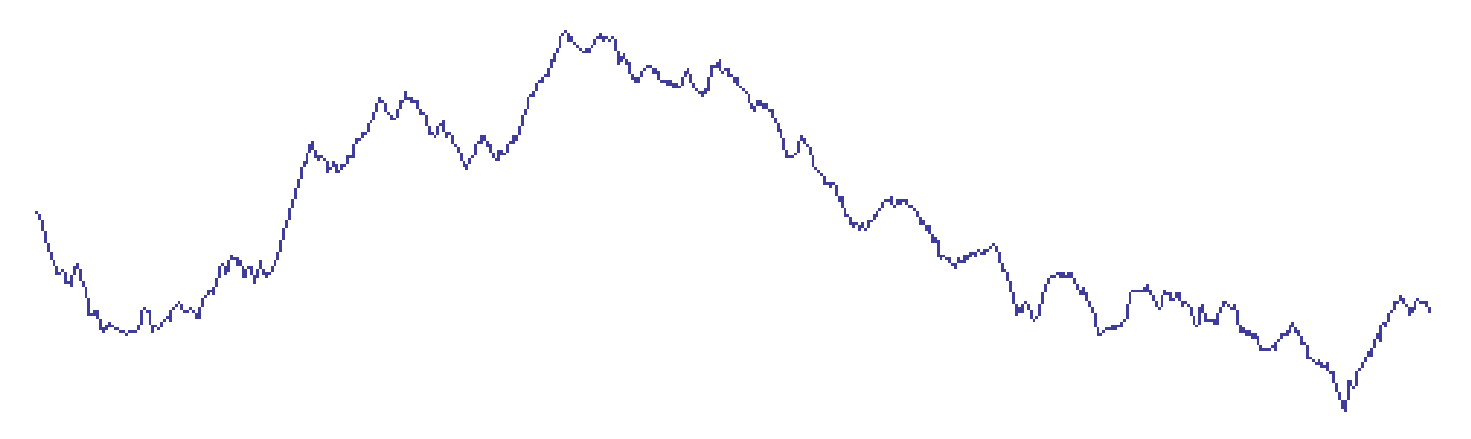

Figure 3: A Fractional Brownian Motion Path with $H=0.7$

the solution process: $d \log S_{t}=\left(r_{f}-\frac{\sigma^{2}}{2}\right) d t+\sigma d B_{t}$, we can discretize it as the follows:

$$
\log S_{t}-\log S_{t-1}=\left(r_{f}-\frac{\sigma^{2}}{2}\right) \Delta t+\sigma \Delta B_{t}
$$

or,

$$
S_{t}=S_{t-1} \exp \left\{\left(r_{f}-\frac{\sigma^{2}}{2}\right) \Delta t+\sigma \Delta B_{t}\right\}
$$

We generate $\Delta B_{t}$ as $\sqrt{t} Z_{i}$, where $Z_{i}$ is an i.i.d. Normal $N(0,1)$. 


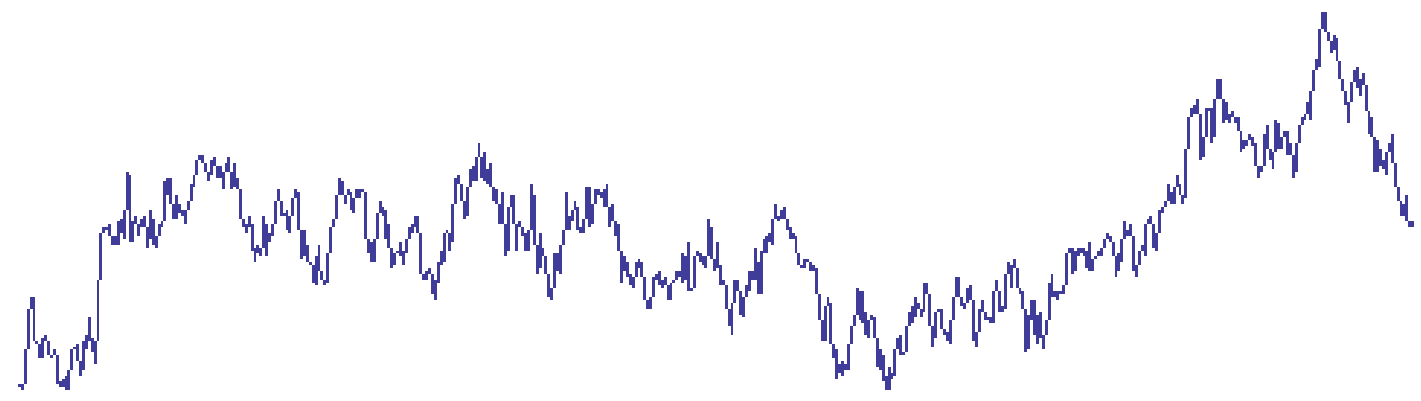

Figure 2: A Fractional Brownian Motion Path with $H=0.3$

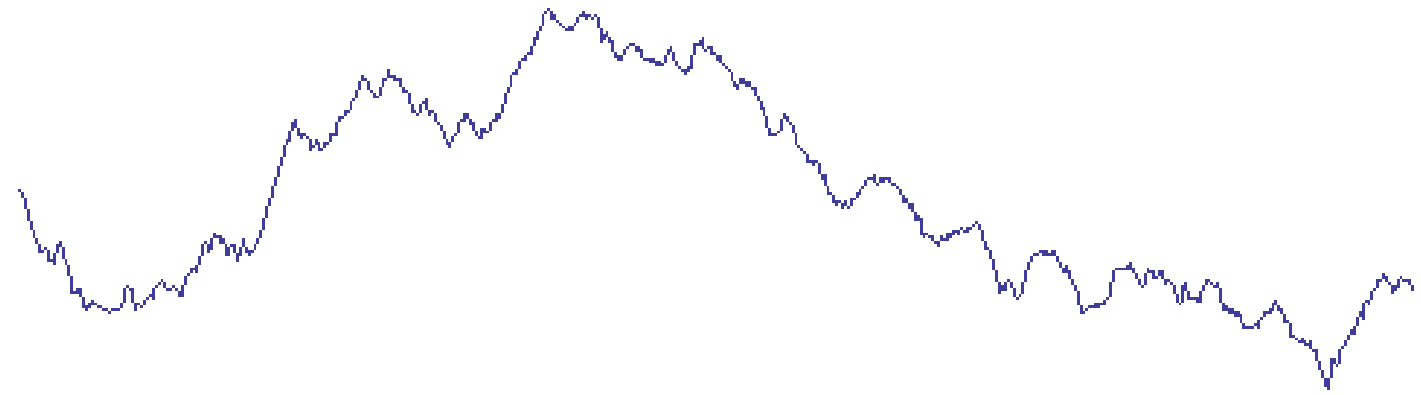

Figure 3: A Fractional Brownian Motion Path with $H=0.7$

the solution process: $d \log S_{t}=\left(r_{f}-\frac{\sigma^{2}}{2}\right) d t+\sigma d B_{t}$, we can discretize it as the follows:

$$
\log S_{t}-\log S_{t-1}=\left(r_{f}-\frac{\sigma^{2}}{2}\right) \Delta t+\sigma \Delta B_{t}
$$

or,

$$
S_{t}=S_{t-1} \exp \left\{\left(r_{f}-\frac{\sigma^{2}}{2}\right) \Delta t+\sigma \Delta B_{t}\right\}
$$

We generate $\Delta B_{t}$ as $\sqrt{t} Z_{i}$, where $Z_{i}$ is an i.i.d. Normal $N(0,1)$. 


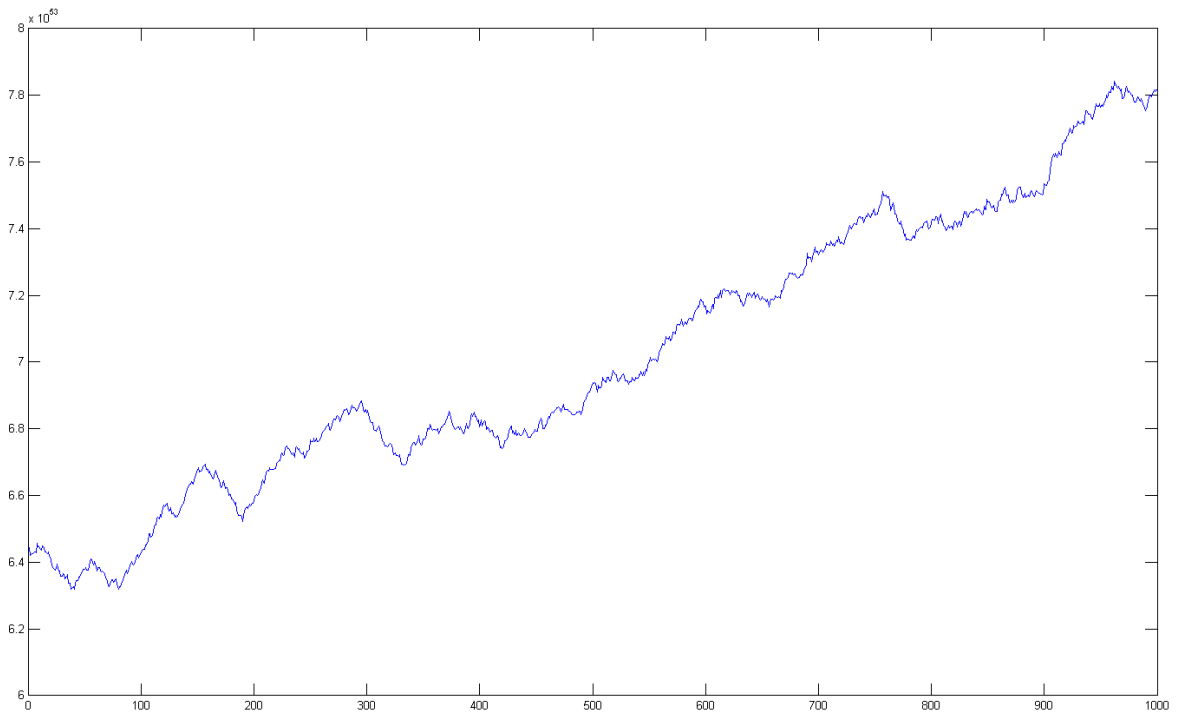

Figure 4: Simulation of a Black-Scholes-Merton Diffusion Process Path

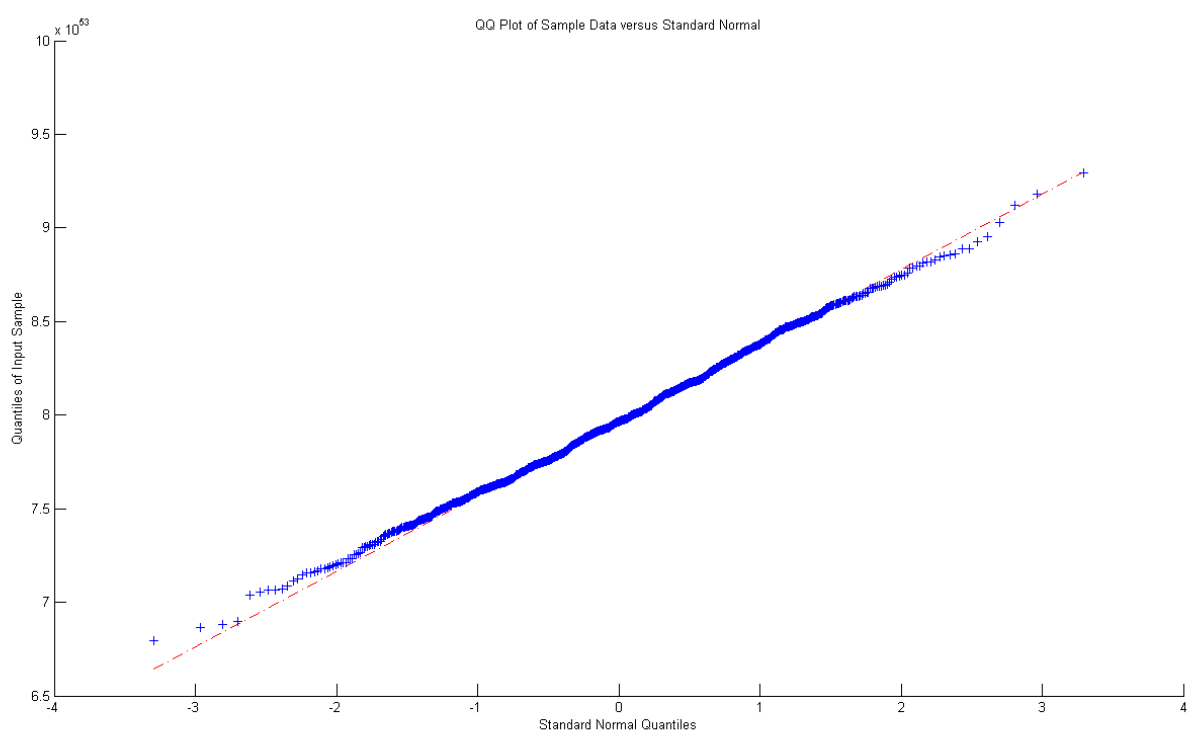

Figure 5: QQ Plot of a Black-Scholes-Merton Diffusion Process 


\section{Simulation Steps:}

1. Decide on the maturity time T.

2. Discretize path into 1000 steps.

3. Then the time increment of each step is $\Delta t=T / 1000$.

4. Generate 1000 i.i.d $Z \sim N(0,1)$ random numbers.

5. Implement iterations $\log S_{t}=\log S_{t-1}+\left(r_{f}-\frac{\sigma^{2}}{2}\right) \Delta t+\sigma \sqrt{\Delta t} Z$ for stock price $S_{i}$.

A resulting Black-Scholes-Merton process path is in Figure 4 and we can see that in Figure 5, the QQ Plot of Black-Scholes model, it is very in line with a Normal distribution, as we expected.

\subsection{Simulation of Merton Jump Diffusion Model}

Recall that the solution of the price process under the Merton Jump Diffusion Model gives:

$$
S(t)=S(0) \exp \left\{\left(\mu-\frac{1}{2} \sigma^{2}\right) t+\sigma B(t)\right\} \prod_{j=1}^{N(t)} Y_{j}
$$

or,

$$
\log S(t)=\log S(0)+\left(\mu-\frac{1}{2} \sigma^{2}\right) t+\sigma B(t)+\sum_{j=1}^{N(t)} \log Y_{j}
$$

\subsubsection{Simulation at Fixed Dates}

We discretize time from 0 to maturity time $\mathrm{T}$ into $\mathrm{n}$ equally spaced time steps, namely, $0=t_{0}<t_{1}<t_{2}<\ldots<t_{n}$, there may be jumps or none during each of these discretized time intervals. 
We denote the $\log$-price process $\log S(t)$ as $X(t)$, hence,

$$
X\left(t_{i+1}\right)=X\left(t_{i}\right)+\left(\mu-\frac{1}{2} \sigma^{2}\right)\left(t_{i+1}-t_{i}\right)+\sigma\left[W\left(t_{i+1}\right)-W\left(t_{i}\right)\right]+\sum_{j=N\left(t_{i}\right)+1}^{N\left(t_{i+1}\right)} \log Y_{j}
$$

\section{Simulation Steps:}

1. Generate Poisson random variable $M=\operatorname{Poisson}\left(\lambda\left(t_{i+1}-t_{i}\right)\right)$

2. Generate standard Normal random variable $Z=N(0,1)$

3. Generate $N$ i.i.d. $\log Y_{j}$

- $Y_{j}$ is $\log$-normal, then $\log Y_{j}=N\left(a, b^{2}\right)$

4. Set iteration $X\left(t_{i+1}\right)=X\left(t_{i}\right)+\left(\mu-\frac{1}{2} \sigma^{2}\right)\left(t_{i+1}-t_{i}\right)+\sigma\left(\sqrt{t_{i+1}-t_{i}} Z\right)+\sum_{j=1}^{M} \log Y_{j}$.

- If $Y_{j}$ is $\log$-normal, then $\sum_{j=1}^{M} \log Y_{j}=N\left(M a, M b^{2}\right)$.

A resulting Merton Jump-Diffusion process path is seen in Figure 6 and we can see that the QQ plot of Merton Jump-Diffusion process in Figure 7 shows obvious fatter tails than a Normal distribution, which is dictinctive from the Black-ScholesMerton process. 


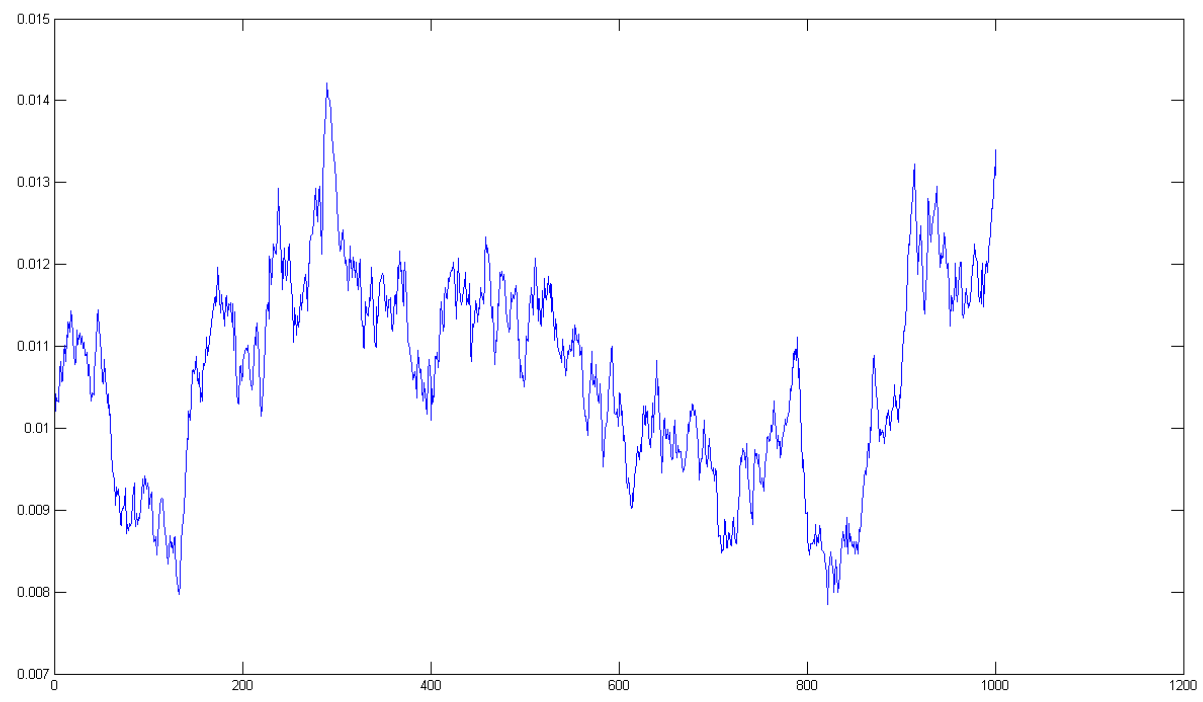

Figure 6: Simulation of a Merton Jump Diffusion Process using Fixed Dates Scheme

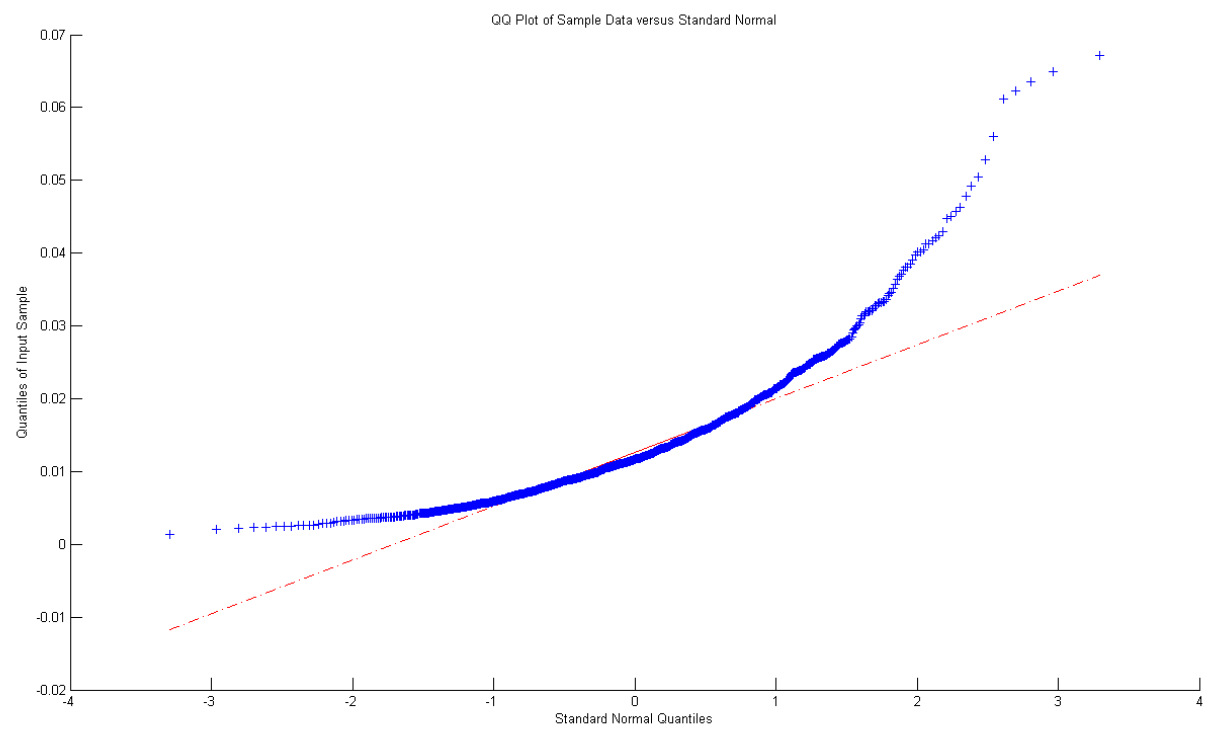

Figure 7: QQ Plot of a Merton Jump Diffusion Process using Fixed Dates Scheme 


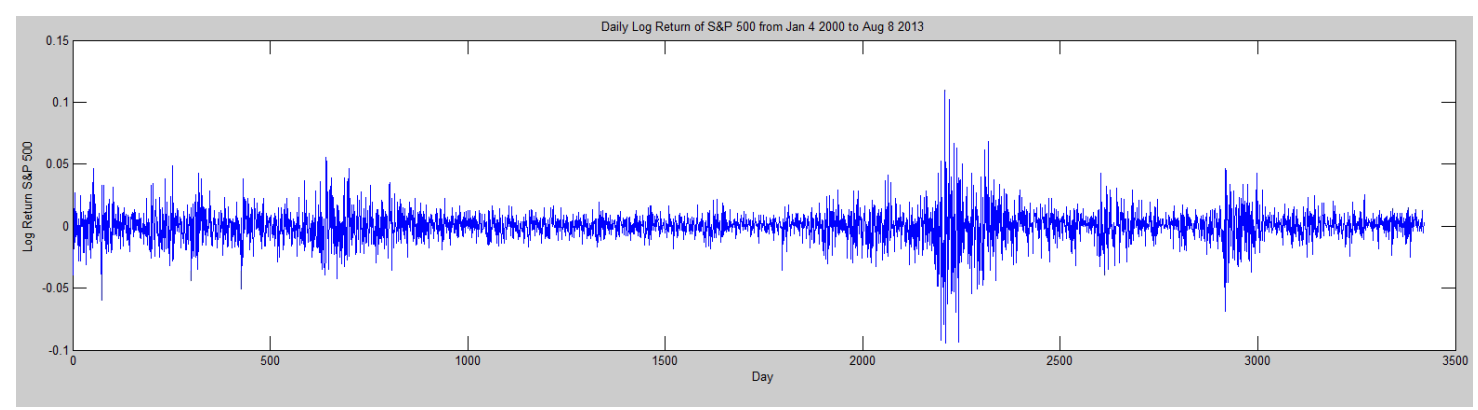

Figure 8: Log Daily Return S\&P 500 Index: from 4 Jan 2000 to Aug 82013

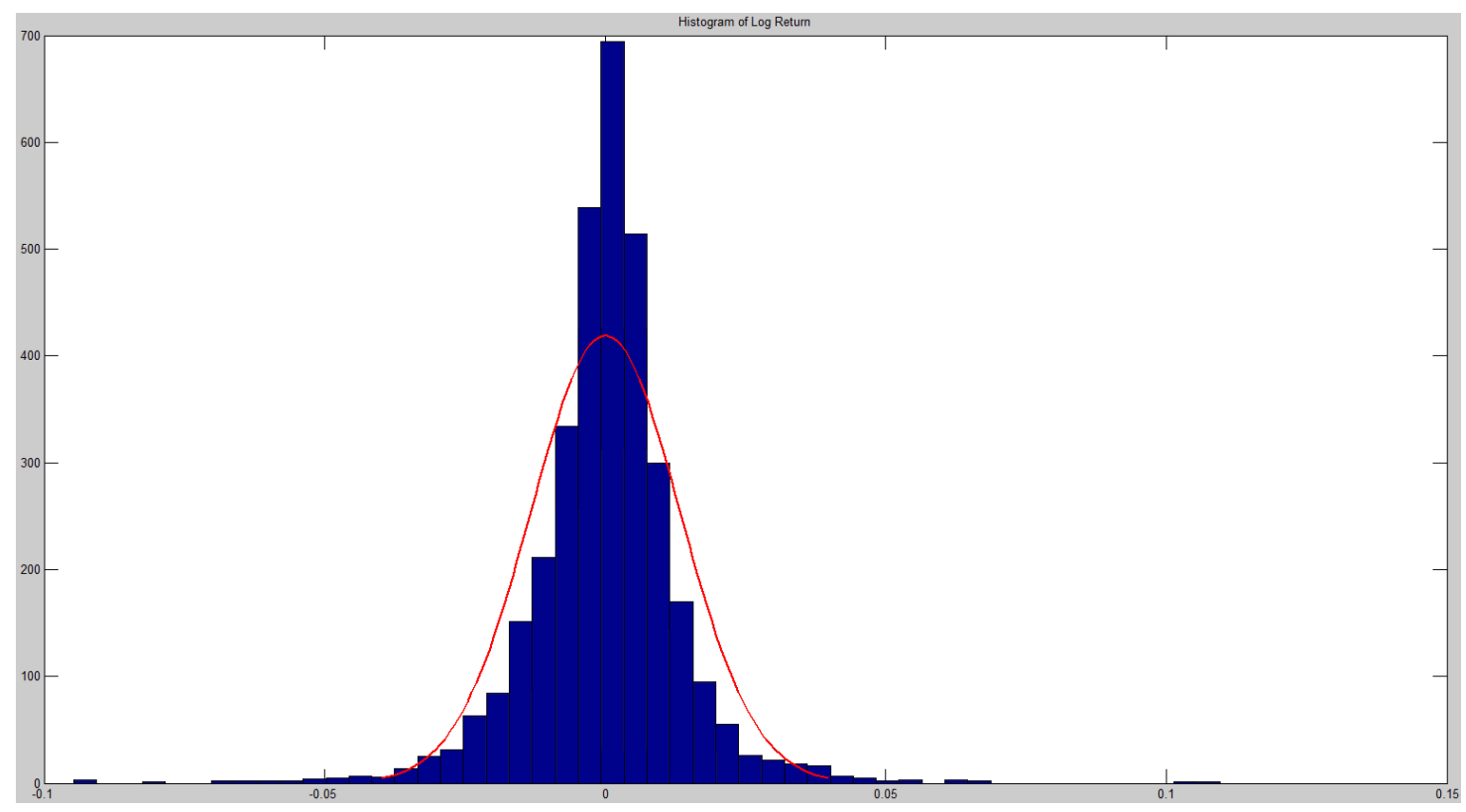

Figure 9: Histogram of Log Return S\&P 500 Index Compared to Standard Normal

\subsection{Empirical Examination of Daily Returns on Standard \& Poor 500 Index}

This section considers the random behaviour of the S\&P 500 daily stock index at adjusted closing price from January 42000 to August 8 2013, the time seires plot and Histogram plot of log return are shown in Figure 8 and 9 . We consider several empirical stylized facts of the $S \& P 500$ log return series in the following as mentioned in Yi's (2010):

\section{Leptokurtic Features:}


1. There are big spikes

2. "Leptokurtic": the histogram displays a high peak and asymmetric fat tails. "Leptokurtic" means the kurtosis of the distribution is large. The kurtosis and skewness are defined as

$$
K=E\left(\frac{(X-\mu)^{4}}{\sigma^{4}}\right), \quad S=E\left(\frac{(X-\mu)^{3}}{\sigma^{3}}\right) .
$$

For normal density, $K=3$. If $K>3$, the distribution will be called leptokurtic and the distribution will have a higher peak and two heavier tails.

In Black-Scholes Brownian Motion model, stock price is modeled as a geometric Brownian Motion,

$$
S(t)=S(0) \exp \{\mu t+\sigma B(t)\}
$$

Brownian Motion $W(t) \sim \mathrm{N}(0, \mathrm{t}), \mu$ represents the drift, measuring the average return, $\sigma$ represents the volatility measuring the standard deviation of the return distribution. Continuous compounded return $\ln (S(t) / S(0))$ has a normal distribution. The Black-Scholes-Merton model does not exhibit fat tails and or mean reversion. For this reason, a number of improved models have been developed, for example the following mentioned in Brigo, Dalessandro, Neugebauer, Triki's (2008), among which, there is the Jump-Diffusion model we described in Section 7.4.1.

\section{Alternative models to Black-Scholes}

Modify BS models to incorporate 3 empirical stylized facts: leptokurtic feature, volatility clustering effect and implied volatility smile.

1. Jump-diffusion models proposed by Merton(1976) and Kou (2002).

$$
S(t)=S(0) \exp \left\{\left(\mu-\frac{1}{2} \sigma^{2}\right) t+\sigma B(t)\right\} \prod_{i=1}^{N(t)} \exp \left\{Y_{i}\right\}
$$

$\mathrm{Y}$ is normal in Merton and double exponential in Kou. The latter enables to get 
analytical solutions for many path-dependent options, including barrier and lookback options and analytical approximations for American options.

2. Chaos theory and fractional Brownian motions Replace Brownian motion by fractional Brownian motion. The latter has dependent increments (rather than independent increments). (Mandelbrot(1963), Rogers(1997) and Salopek (1998) show that these models may lead to arbitrage opportunities). Consistent price systems for the case of fractional Brownian motion when transaction costs are included are deiscussed in Guasoni, Rasonyi and Schachermayer (2008).

3. Generalized hyperbolic models, including log t model and log hyperbolic model, and stable processes to replace the normal distribution assumption by some other distributions. (Barndorff-Nielsen and Shephard(2001), Samorodnitsky and Taqqu(1994), Blattberg and Gonedes(1974)).

4. Models based on Lévy processes (Cont and Tankov 2004) and reference therein.

5. Stochastic volatility and GARCH models (Hull and White 1987), Engle 1995, Fouque et al. 2000, Heston 1993. These models are to capture the volatility clustering effect.

6. Time changed Brownian Motions and time changed Lévy processes. Asset price $S(t)$ is modeled as

$$
S(t)=G(M(t))
$$

G is either geometric BM or a Lévy. $\mathrm{M}(\mathrm{t})$ is a nondecreasing stochastic process modeling the stochastic activity time in the market. that may link to trading volumes. Clark 1973, Madan and Seneta 1990 Madan et al. 1998, Heyde 2000, Carr et al. 2003. 
7. Affine stochastic-volatility and affine jump-diffusion, Duffie et al. 2000, which combines both stochastic volatilities and jump-diffusions. 


\section{Appendices}

\section{A Modes of Convergence}

\section{A.1 Mean Square Convergence}

As introduced in Thomas Mikosch's (1998),

Definition A.1 we say a sequence $\left\{A_{n}\right\}$ converges in mean square to $A$ if

$E\left[\left|A_{n}\right|^{2}+|A|^{2}\right]<\infty$ for all $n$ and

$$
E\left[\left|A_{n}-A\right|^{2}\right] \rightarrow 0
$$

We denote this convergence as $A_{n} \rightarrow L^{L^{2}} A$.

If it was not 2 , but a general $p>0$, we call it a $L^{p}$ - Convergence or convergence in p-th mean.

Theorem A.1 Convergence in $p$-th mean implies convergence in probability.

Proof. $P\left\{\left|X_{n}-X\right|>\epsilon\right\} \leq \frac{E\left|X_{n}-X\right|^{p}}{\epsilon^{p}}$. Therefore, when taking limits on both sides, if the right hand side converges to 0 (convergence in $p-t h$ mean), the left hand side also goes to 0 (convergence in probability).

Theorem A.2 Convergence in probability implies convergence almost surely along a subsequence. 
PROOF. Assume $X_{n}$ converges to 0 in probability, we want to prove that a subsequence $n_{k}$ exists such that $X_{n_{k}}$ converges to 0 almost surely.

$$
\begin{aligned}
X_{n} \rightarrow^{p} 0 & \Rightarrow \text { for } \forall k>0, \lim _{n \rightarrow \infty} P\left(\left|X_{n}\right|>\frac{1}{2^{k}}\right)=0 \\
& \Rightarrow \text { for } \forall k>0, \exists n_{k} \text { s.t. } P\left(\left|X_{n_{k}}\right|>\frac{1}{2^{k}}\right)<\frac{1}{2^{k}} \\
& \Rightarrow \sum_{k} P\left(\left|X_{n_{k}}\right|>\frac{1}{2^{k}}\right)<\sum_{k} \frac{1}{2^{k}}<\infty
\end{aligned}
$$

If we denote $E_{k}=\left\{\left|X_{n_{k}}\right|>\frac{1}{2^{k}}\right\}$, then $P\left(\left|X_{n_{k}}\right|>\frac{1}{2^{k}}\right.$ i.o. $)=0$, in other words, we have such a subsequence $n_{k}$ such that $X_{n_{k}} \rightarrow^{\text {a.e. }} 0$.

\section{B Function Approximation and Convergence in $\mathcal{L}^{2}$-Norm}

\section{B.1 Sufficient Conditions for Convergence in $\mathcal{L}^{2}$}

Theorem B.1 Cauchy sequence in $\mathcal{L}^{2}$ converges in $\mathcal{L}^{2}$-Norm to a unique limit.

\section{B.2 Function Approximation in $\mathcal{L}^{2}$-Norm}

Theorem B.2 Non-negative functions in $\mathcal{L}^{2}$ can be approximated by a monotonically increasing sequence of non-negative simple measurable functions in $\mathcal{L}^{2}$-norm. Namely, If $f \geq 0$ and $f \in \mathcal{L}^{2}$, we can find $\left\{s_{n}\right\} \rightarrow{ }^{L^{2}} f$ s.t.

- $s_{n}$ is simple measurable, $s_{n} \geq 0$ and increasing

- $\left\|f-s_{n}\right\|_{\mathcal{L}^{2}} \rightarrow 0$

And the general function case follows.

The above theorem can be found in Rudin's (1964) book. 
C FUNCTION APPROXIMATION AND CONVERGENCE IN EUCLIDEAN NORM69

\section{Function Approximation and Convergence in Euclidean} Norm

Theorem C.1 A real-valued function on a measure space can be approximated by a sequence of simple functions in Euclidean norm. Here a simple function is defined to be a function with finite range. Namely, this says:

If $f \in \mathcal{R}$, then we can find $\left\{s_{n}\right\} \rightarrow f, \forall s \in X$ s.t.

- $\left|s_{n}-f\right| \rightarrow 0, \forall x \in X$

- if $f \geq 0$, then $\left\{s_{n}\right\}$ can be monotonically increasing non-negative

- iff measurable, $\left\{s_{n}\right\}$ can be measurable.

The above theorem can be found in Rudin (1964) (10.20).

\section{From Euclidean Convergence to $\mathcal{L}^{2}$ Convergence}

\section{Theorem D.1 DCT - Dominated Convergence Theorem}

If $f_{n}$ is a sequence of measurable function that converges pointwise to $f$ in Euclidean Norm, then it converges to $f$ in $\mathcal{L}^{2}$-Norm if:

- there exists a measurable function $g$ that dominates $f_{n}$ by $\left|f_{n}\right|^{2}<g, \forall x$ and $\forall n$ and $\int g(x) d \mu<\infty$.

By pointwise convergence, we mean $\left|f_{n}-g\right| \rightarrow 0$ for all $x$.

By $\mathcal{L}^{2}$ Convergence, we mean $\left\|f_{n}-f\right\|_{\mathcal{L}^{2}} \rightarrow 0$.

In general it is also true for $\mathcal{L}^{1}$ convergence. 


\section{E Adapted Stochastic Processes}

The following definitions in this section can be seen in Shreve (2004). Firstly, we need to define a $\sigma$-algebra generated by a random variable.

Definition E.1 Given $X$ as a random variable defined on a nonempty sample space $\Omega$, $X: \Omega \rightarrow \mathbb{R}$, the $\sigma$-algebra generated by $X$, denoted by $\sigma(X)$, is the collection of all subsets of $\Omega$ of the form $\{\omega \in \Omega: X(\omega) \in B\}$, where $B$ ranges over the Borel subsets of $\mathbb{R}$

Secondly, we need to define a random variable which is $\mathcal{F}$-measurable.

Definition E.2 Given $X$ as a random variable defined on a nonempty sample space $\Omega$, $X: \Omega \rightarrow \mathcal{R}$. Let $\mathcal{F}$ be a $\sigma$-algebra of subsets of $\Omega$. If every set in $\sigma(X)$ is also in $\mathcal{F}$, we say that $X$ is $\mathcal{F}$-measurable. In other words, $\sigma(X) \subset \mathcal{F}$.

Definition E.3 Given a nonempty sample space $\Omega$ equipped by a filtration $\mathcal{F}(t)$ for $t \in$ $[0, T]$. Let $X(t)$ be a collection of random variables indexed by $t$. This collection of random variables are called an adapted stochastic process if, for each $t$, the random variable $X(t)$ is $\mathcal{F}(t)$-measurable. 


\section{References}

[1] Biagini, Francesca; Hu, Yaozhong; Øksendal, Bernt; Zhang, Tusheng (2008), Stochastic Calculus for Fractional Brownian Motion and Applications, Springer.

[2] Brigo, Damiano; Dalessandro, Antonio; Neugebauer, Matthias; Triki, Fares (2008), A Stochastic Processes Toolkit for Risk Management.

Fitch Solutions, Fitch Ratings, Paris School of Economics and Department of Mathematics, Imperial College, London.

[3] Brzezniak, Zdzislaw; Zastawniak, Tomasz (1999), Basic Stochastic Processes, Springer.

[4] Chorro, Christophe (2008), Monte Carlo Methods and Black Scholes model. l’Université Paris 1.

[5] Chung, Kai Lai (2001), A Course in Probability Theory. Third Edition, Academic Press.

[6] Coelen, Nathan (2002), Black-Scholes Option Pricing Model.

[7] Cook, John D. (2003), Notes on hypergeometric functions.

[8] Feldman, Joel (2008), Notes: Math 321 Real Variables II. Mathematics Department, University of British Columbia.

[9] Guasoni, P., Rasonyi, M. and Schachermayer, W. (2008), Consistent price systems and face-lifting pricing under transaction costs. Annals of Applied Probability 18, 491-520.

[10] Glasserman, Paul (2003), Monte Carlo Methods in Financial Engineering, Springer. 
[11] Glynn, Peter, Notes: MSEE 322 Stochastic Calculus and Control. Stanford University.

[12] Haugh, Martin, Simulating Stochastic Differential Equations

Monte Carlo Simulation: IEOR E4703 (C)2004.

[13] Hull, John (1997), Introduction to Futures and Options Markets.

Prentice Hall, Inc.

[14] Junge, Marius (2004), Notes: Math 540 Real Analysis.

University of Illinois at Urbana-Champaign.

[15] Karatzas, and Shreve (1988), Brownian Motion and Stochastic Calculus. Springer-Verlag(1988).

[16] Kawada T., and Kôno N. (1973), On the variaion of Gaussian Processes. Lecture Notes in Mathematics.

[17] Kijima, Masaaki and Tam Chun Ming (2013), Theory and Applications of Monte Carlo Simulations.

InTech

[18] Kozdron, Michael (2007), Notes: Statistics 862 - Stochastic Processes. University of Regina.

[19] Labute, John (2003), Notes: MATH 255 Honours Analysis 2. McGill University.

[20] Matsuda, Kazuhisa, Introduction to Merton Jump Diffusion Model. Department of Economics, The Graduate Center, The City University of New York.

[21] Mikosch, Thomas (1998), Elementary Stochastic Calculus with Finance in View. World Scientific. 
REFERENCES

[22] Øksendal, Bernt (2003), Stochastic Differential Equations - An Introduction with Applications Sixth Edition, Springer.

[23] Olson, Luke (2009), Notes: Integration: Gauss Quadrature.

Department of Computer Science, University of Illinois at Urbana-Champaign.

[24] Privault, Nicolas, Notes on Stochastic Finance.

Division of Mathematical Sciences, School of Physical and Mathematical Sciences, Nanyang Technological University, Singapore.

[25] Rudin, Walter (1964), Principles of Mathematical Analysis, Second Edition. McGraw-Hill Book Company.

[26] Shreve, Steven (2004), Stochastic Calculus for Finance:v.2 Continuous-time Models. Springer-Verlag New York Inc.

[27] Sarkar, Soumik (2011), Fractional Brownian Motion: Long range dependency, Markov property, Simulation strategy A review.

Pennsylvania State University.

[28] Salopek, D.M. (1998), Tolerance to arbitrage.

Stochastic Processes and their Applications 76 (1998) 217-230.

[29] Taylor, S.J. (1972), Exact asymptotic estimates pf Brownian path variation, Duke Math. Journal(1972), 210-241.

[30] Visser, Marcel (2009), Volatility Proxies and GARCH models, Dissertation University of Amsterdam.

[31] Yi, Linghao (2010), The pricing of Options with Jump Diffusion and Stochastic Volatility. Copyright (C)2010 Faculty of Industrial Engineering, Mechanical Engineering and Computer Science, School of Engineering and Natural Sciences, University of Iceland. 
[32] Young, L.C. (1936), An inequality of Hölder connected with Stieltjes integration, Acta Math. 67(1936). 251-282. 